\title{
Discrete spectral symmetries of low-dimensional differential operators and difference operators on regular lattices and two-dimensional manifolds
}

\author{
S. P. Novikov and I. A. Dynnikov*
}

\section{Contents}

$\S 1$. Introduction: history of the problem, the one-dimensional Schrödinger operator

§2. The non-stationary one-dimensional Schrödinger equation 6

§3. One-dimensional difference operators 15

\$4. The Laplace transformation and the two-dimensional Schrödinger $\begin{array}{ll}\text { operator in a magnetic field } & 18\end{array}$

§5. Difference equations and Laplace transformations. The hyperbolic case

86. The Laplace transformation for elliptic two-dimensional difference operators on a regular lattice. Equations of a triangle, curvature

\$7. Factorizations and Laplace transformations on many-dimensional $\begin{array}{ll}\text { lattices of regular tetahedra in } \mathbb{R}^{\mathrm{N}} & 48\end{array}$

§8. Factorizations of operators and Laplace transformations on \begin{tabular}{l|ll} 
two-dimensional surfaces & 53
\end{tabular}

§9. Simplicial connections. Generalizations 56

*The work of the second author was partially supported by the Russian Foundation for Fundamental Research (grant no. 96-01-01404). The last three sections of the survey present the joint work of the authors announced in 1 . 


\section{$\S 1$. Introduction: history of the problem, the one-dimensional Schrödinger operator}

Already in the 18th century (1742) Euler paid attention to the substitutions which transform solutions of a certain ordinary linear differential equation into solutions of another equation associated with it. Let

$$
L=-\partial_{x}^{2}+u(x)
$$

be the Sturm-Liouville operator defined on the real line $(x)$, and let $\psi(x, \lambda)$ be a solution of the linear equation $L \psi=\lambda \psi$. Let $v(x)$ be an arbitrary solution of the

Riccati equation

$$
u(x)=v_{x}+v^{2} .
$$

Under these conditions we have the following result.

Lemma 1 The new function $\widetilde{\psi}=\psi_{x}-v(x) \psi$ satisfies the new equation $\widetilde{L} \widetilde{\psi}=$ $\lambda \widetilde{\psi}$, where

$$
\widetilde{L}=-\partial_{x}^{2}+\widetilde{u}(x), \quad \widetilde{u}(x)=-v_{x}+v^{2} .
$$

Proof This lemma easily follows from the fact that $L$ is in fact the product of two non-commuting factors of the first order:

$$
L=-\partial_{x}^{2}+u=-\left(\partial_{x}+v\right)\left(\partial_{x}-v\right),
$$

where $u=v_{x}+v^{2}$. Further, we have

$$
-\psi_{x x}+u \psi=\lambda \psi=-\left(\partial_{x}+v\right)\left(\partial_{x}-v\right) \psi=-\left(\partial_{x}+v\right) \widetilde{\psi} .
$$

Hence,

$$
\widetilde{L} \widetilde{\psi}=-\left(\partial_{x}-v\right)\left(\partial_{x}+v\right) \widetilde{\psi}=\lambda\left(\partial_{x}-v\right) \psi=\lambda \widetilde{\psi}
$$

where

$$
\widetilde{L}=-\left(\partial_{x}-v\right)\left(\partial_{x}+v\right)=-\partial_{x}^{2}+\widetilde{u}(x) .
$$

We have proved Lemma 1.

Hence, the Euler substitution is based on the representation of $L$ as the product of first-order operators which are formally adjoint to each other if $u, v \in$ $\mathbb{R}:$

$$
L=-\left(\partial_{x}+v\right)\left(\partial_{x}-v\right)=Q Q^{+},
$$

and is given by interchanging them:

$$
Q Q^{+}=L \longmapsto \widetilde{L}=Q^{+} Q, \quad \psi \longmapsto \widetilde{\psi}=Q^{+} \psi .
$$

In the literature the Euler substitutions are often called 'Darboux transformations' or 'Bäcklund-Darboux transformations' because of the role they 
play in the theory of non-linear systems of Korteweg-de Vries (KdV) type for functions $u(x, t)$ that depend on the time as the parameter.

The classical mathematicians put it like this: the Darboux transformation $L \mapsto \widetilde{L}$ is determined by one chosen solution $\varphi$ of the equation $L \varphi=0$, and

$$
\widetilde{\psi}=\psi_{x}-\left(\varphi_{x} / \varphi\right) \psi
$$

We have

$$
v(x)=\varphi_{x} / \varphi, \quad \text { where } \quad v_{x}+v^{2}=u(x) .
$$

The algebraic language, that is, the correspondence of this with the factorization $\alpha+L=Q Q^{+}$, was used after the works of physicists of the 30s and 40s (Dirac, Schrödinger, Infeld).

¿From the point of view of formal spectral theory, that is, of local solutions of the equation $L \psi=\lambda \psi$, the Darboux-Euler transformation generates a transformation of the eigensubspaces for all $\lambda$ :

$$
B_{\lambda} \psi \longmapsto \widetilde{\psi},
$$

where $L \psi=\lambda \psi, \widetilde{L} \widetilde{\psi}=\lambda \widetilde{\psi}, \widetilde{\psi}=\psi_{x}-v \psi$.

We should talk about the Darboux-Euler transformation of the whole linear hull (with respect to all $\lambda$ )

$$
B \sum a_{i} \psi_{i} \longmapsto \sum a_{i} \widetilde{\psi}_{i}
$$

where $L \psi_{i}=\lambda_{i} \psi_{i}$ and $a_{i}$ are arbitrary coefficients.

The transformation $B$ is not, strictly speaking, an isomorphism: for $\lambda=0$ and $\psi=\varphi$ we have $B \varphi=0, v=\varphi_{x} / \varphi$. Hence, the kernel of this transformation is one-dimensional.

Turning to the present 'global' spectral theory of the operator $L$ in the Hilbert space $\mathcal{L}_{2}(\mathbb{R})$, we see that if the function $\varphi(x)$ does not belong to $\mathcal{L}_{2}(\mathbb{R})$, then the Darboux transformation $B$ can generate an isomorphism of the spectral theories of the operators $L$ and $\widetilde{L}$; sometimes $B$ is a monomorphism on a part of the spectrum of $L$ that covers all but one eigenfunction in $\mathcal{L}_{2}(\mathbb{R})$; sometimes (if $\varphi(x) \neq 0$ and $\left.\varphi \in \mathcal{L}_{2}(\mathbb{R})\right) B$ annihilates exactly one state of the spectrum $\varphi$, the lowest one, the 'basic' one in $\mathcal{L}_{2}(\mathbb{R})$. We do not know the complete classification of all possible cases from the point of view of spectral theory in $\mathcal{L}_{2}(\mathbb{R})$.

Definition 1 We call the Darboux-Euler transformation the latent algebraic symmetry of the spectral theory of the one-dimensional Schrödinger operator, or the discrete symmetry.

We recall that the 'discontinuous spectral symmetries' or 'isospectral deformations' of the operator $L$ are the well-known systems of the theory of solitons like $\mathrm{KdV}$, which generate a gigantic commutative continuous group and in which $\mathrm{KdV}$ is a one-parameter subgroup, see [2], [3].

The Darboux-Euler transformation depends on a parameter, which appears in the factorization (3), that is, on the choice of a solution of the Riccati equation (2). We denote these transformations by $B$,

$$
B L \longmapsto \widetilde{L}=-\left(\partial_{x}-v\right)\left(\partial_{x}+v\right), \quad \psi \longmapsto \widetilde{\psi}=\left(\partial_{x}-v\right) \psi .
$$


Moreover, there is still a one-parameter family of transformations of 'energy shift'

$$
T_{\alpha} L \longmapsto L+\alpha, \quad \alpha=\text { const. }
$$

Let $B_{\alpha}=B \circ T_{\alpha}$.

In the theory of solitons, by means of the transformations $B_{\alpha}$ we could create well-known non-reflecting potentials from the zero potential. By one application of the transformation $B_{\alpha}$ we can obtain 'soliton potentials' from the free operator $L_{0}=-\partial_{x}^{2}$, starting with $v=k \tanh k\left(x-x_{0}\right), \varphi=\cosh k\left(x-x_{0}\right), \alpha=k^{2}$. By multiple application of the transformations $B_{\alpha_{j}}$ we can obtain all rapidly decreasing non-reflecting potentials, taking each time a non-vanishing solution $\varphi_{j}$

'below the spectrum':

$$
\begin{gathered}
u_{N+1}(x)=B_{\alpha_{N}} \circ B_{\alpha_{N-1}} \circ \cdots \circ B_{\alpha_{0}}(0), \\
u_{0}(x)=0, u_{1}(x)=-2 k^{2} / \cosh ^{2} k\left(x-x_{0}\right), \ldots .
\end{gathered}
$$

In the literature on the theory of solitons (see [3]-[5]), by means of BäcklundDarboux transformations the so-called 'many-soliton potentials on the background of finite-zone potentials' were obtained by applying the transformations $B_{\alpha}$ with suitable parameters to the known periodic and quasiperiodic finitezone potentials. This class of potentials corresponds to the limits of finite-zone potentials for various degenerate Riemann surfaces. For a long time nobody succeeded in establishing the relation between the class of non-degenerate periodic (quasiperiodic) finite-zone potentials (one-dimensional Schrödinger operators) and the theory of Bäcklund-Darboux transformations. We recall that finite-zone potentials correspond to orbits of the infinite-dimensional continuous commutative group of isospectral symmetries, the so-called higher analogues of $\mathrm{KdV}$, which have finite dimension ([2], [6]-[11]). These operators have remarkable spectral and algebro-geometric properties: their coefficients are calculated by means of theta-functions of hyperelliptic Riemann surfaces, and they generate remarkable fully integrable Hamiltonian systems.

An important idea on the connection between Bäcklund-Darboux transformations and the theory of finite-zone potentials was proposed in 1986 ([12]). In this work the author considered 'cyclic chains' of the Bäcklund-Darboux transformations of length $N+1$, that is, equations of the form

$$
u=B_{\alpha_{N}} \circ B_{\alpha_{N-1}} \circ \cdots \circ B_{\alpha_{0}}(u)
$$

under the condition $\alpha_{N}=\alpha_{N-1}=\cdots=\alpha_{0}=0$. Developing very interesting technical arguments in studying such chains as certain integrable non-linear systems, in [12] the supposition was put forward that for $N=2 k$ the potential $u(x)$ is always a finite-zone potential (Weiss' hypothesis). This hypothesis was proved in [13] in a more general form.

Suppose that $N=2 k$ and the $\alpha_{j}$ may be non-zero. If $\sum_{j=0}^{N} \alpha_{j}=0$, then the potential $u(x)$ in the cyclic chain of Bäcklund-Darboux transformations is finite-zone with a Riemann surface of genus no greater than $N+1$. 
If $\sum_{j=0}^{N} \alpha_{j}=\alpha \neq 0$, then the potential $u(x)$ has an 'oscillator-similar' asymptotic form

$$
u(x)=\frac{\alpha^{2} x^{2}}{4(N+1)^{2}}+O(x), \quad|x| \rightarrow \infty .
$$

If the potential $u(x)$ is smooth and real (without singularities), then the spectrum of the operator $L=-\partial_{x}^{2}+u(x)$ is a combination of $N+1$ arithmetic progressions with the general difference $\alpha$. At the same time there is a differential operator $A_{N+1}$ of order $N+1$ such that $\left[A_{N+1}, L\right]=\alpha A_{N+1}$.

V. E. Adler made a numerical investigation and established that the equation of cyclicity of the chains of transformations $B_{\alpha_{j}}$ for $\alpha \neq 0$ has non-singular real solutions. In particular, for $N=2$ this equation is transformed into the Painlevé-IV equation.

Operators of the cyclic chain

$$
L_{0}=L_{N+1}, L_{N}, \ldots, L_{1}, L_{0}, \ldots
$$

have factorizations of the form $\alpha_{j}+L_{j}=Q_{j} Q_{j}^{+}$. ¿From the definition of Darboux-Euler substitutions we have:

$$
\begin{array}{r}
L_{j}+\alpha_{j}=Q_{j} Q_{j}^{+}=-\left(\partial_{x}+v_{j}\right)\left(\partial_{x}-v_{j}\right), \\
L_{j+1}=\widetilde{L}_{j}=Q_{j}^{+} Q_{j} .
\end{array}
$$

For the operators $L_{j}$ we consider a set of 'ground states' defined by the equation

$$
Q_{j-1} \psi_{0, j}=0=L_{j} \psi_{0, j} .
$$

We assume that $\psi_{0, j} \in \mathcal{L}_{2}(\mathbb{R})$, and all the translations $\alpha_{j}$ and the coefficients of the operators $Q_{j}, Q_{j}^{+}$are real. Then the sequence of 'creation operators' gives the whole spectrum in $\mathcal{L}_{2}(\mathbb{R})$ for operators $L_{M}(M \geq j)$ according to the formula

$$
\begin{aligned}
L_{M} \psi_{M-j, j} & =\left(\sum_{K=j}^{M} \alpha_{K}\right) \psi_{M-j, j}, \\
Q_{M}^{+} Q^{+} \ldots Q_{j+1}^{+} Q_{j}^{+} \psi_{0, j} & =\psi_{M-j, j}, \quad M \geq j .
\end{aligned}
$$

Here $L_{M}=L_{M+l(N+1)}, n=2 k, l \in \mathbb{Z}$.

We note that the sequence of numbers $\sum_{K=j}^{M} \alpha_{K}$ is a combination of finitely many arithmetic progressions with common difference $\sum_{K=0}^{N} \alpha_{K}=\alpha$, since the set of numbers $\alpha_{j}$ is periodic. The spectrum of the operator $L_{M}$ has the form $\sum_{K=j}^{M} \alpha_{K}=\lambda_{j, M}, j=M, M-1, M-2, \ldots$.

These are the basic results in the theory of cyclic Bäcklund-Darboux chains for the one-dimensional continuous Schrödinger operator (13]). T It would not be superfluous to see in the literature a publication containing a full justification of this beautiful algebraic picture from the viewpoint of rigorous spectral

\footnotetext{
${ }^{1}$ For non-trivial cyclic chains of even length a solution of the problem of classification has not been obtained.
} 
theory, that is, functional analysis. So far, this problem can be considered 'more or less solved' on the level of the requirements of reasonable (not superrigorous) mathematical physics. An understanding of what happens and a set of rigorous formulae have already been obtained, but the rigorous completeness of the picture has not been proved in general.

Example 1 Let $N=0$ (a cyclic chain of period 1); we have

$$
\cdots=L_{-1}=L_{0}=L_{1}=\cdots,
$$

where $L_{0}=Q^{+} Q, \alpha+L_{1}=Q Q^{+}$. Here there arises the Heisenberg algebra

$$
Q Q^{+}=Q^{+} Q+\alpha, \quad Q=\partial_{x}+\frac{\alpha x}{2} .
$$

Since $Q=\partial_{x}+v(x)$, we have

$$
u(x)=v_{x}+v^{2}=\frac{\alpha}{2}+\left(\frac{\alpha}{2}\right)^{2} .
$$

The eigenfunctions $\psi_{M-j, j}$ (mentioned above) are the eigenfunctions of the quantum oscillator

$L=-\partial_{x}^{2}+\frac{\alpha^{2} x^{2}}{4}=-\left(\partial_{x}+\frac{\alpha x}{2}\right)\left(\partial_{x}-\frac{\alpha x}{2}\right)-\frac{\alpha}{2}=-\left(\partial_{x}-\frac{\alpha x}{2}\right)\left(\partial_{x}+\frac{\alpha x}{2}\right)+\frac{\alpha}{2}$.

Let $\alpha>0$. The equation $Q \psi_{0}=0$ has a solution $\psi_{0} \in \mathcal{L}_{2}(\mathbb{R}), \psi_{0}=e^{-\alpha x^{2} / 4}$. All eigenfunctions $\psi_{M}=\left(Q^{+}\right)^{M} \psi_{0}, Q^{+}=-\left(\partial_{x}-\alpha x / 2\right)$, belong to $\mathcal{L}_{2}(\mathbb{R})$ and generate the spectrum of the operator $L=-\partial_{x}^{2}+\alpha^{2} x^{2} / 4$,

$$
\lambda_{M}=\frac{\alpha}{2}+M \alpha
$$

In this example everything is clear, but we should establish in the general case that the eigenfunctions $\psi_{M-j, j}$ belong to $\mathcal{L}_{2}(\mathbb{R})$.

\section{$\S 2$. The non-stationary one-dimensional Schrödinger equation}

The Darboux substitution for the non-stationary Schrödinger equation

$$
i \psi_{t}=-\psi_{x x}+u \psi
$$

is worthy of mention (see [14]). These substitutions have been used to construct some exact solutions of the KP equation. Starting from an exact solution

$$
i \varphi_{t}=-\varphi_{x x}+u(x, t) \varphi,
$$

we define the transformation

$$
B \psi \longmapsto \widetilde{\psi}=\psi_{x}-\frac{\varphi_{x}}{\varphi} \psi
$$


The function $\widetilde{\psi}$ satisfies the equation

$$
i \widetilde{\psi}_{t}=-\widetilde{\psi}_{x x}+\widetilde{u}(x, t) \widetilde{\psi},
$$

where $\widetilde{u}=u-2(\log \varphi)_{x x}$.

We express this in algebraic language.

Lemma 2 Suppose there is given a real connection of zero curvature

$$
\nabla_{t}=\partial_{t}-w, \quad \nabla_{x}=\partial_{x}-v, \quad\left[\nabla_{t}, \nabla_{x}\right]=0
$$

such that (10) has the form

$$
i \nabla_{t} \psi=\nabla_{x}^{+} \nabla_{x} \psi, \quad \nabla_{x}^{+}=-\left(\partial_{x}+v\right) .
$$

Then the Darboux transformation has the form

$$
\widetilde{\psi}=\nabla_{x} \psi .
$$

The new function $\widetilde{\psi}(x, t)$ satisfies the equation

$$
i \nabla_{t} \widetilde{\psi}=\nabla_{x} \nabla_{x}^{+} \widetilde{\psi}
$$

Proof ¿From (15) it follows that

$$
i \nabla_{t} \psi=\nabla_{x}^{+} \widetilde{\psi}
$$

We apply $\nabla_{x}$ to both sides of this equation and use the relation of zero curvature $\nabla_{x} \nabla_{t}=\nabla_{t} \nabla_{x}$. We obtain

$$
i \nabla_{x} \nabla_{t} \psi=\nabla_{x} \nabla_{x}^{+} \widetilde{\psi}=i \nabla_{t}\left(\nabla_{x} \psi\right)=i \nabla_{t} \widetilde{\psi} .
$$

We have proved Lemma 2 .

The representation (15) is determined by one solution $\varphi$ of (11), since the connection of zero curvature has the form

$$
\nabla_{t}=\partial_{t}-\frac{\varphi_{t}}{\varphi}, \quad \nabla_{x}=\partial_{x}-\frac{\varphi_{x}}{\varphi} .
$$

Hence, a substitution of Euler-Darboux type here hardly differs from the case of the stationary Schrödinger operator.

We now consider cyclic chains of length 1 . Let the Schrödinger equation and a chosen solution $\varphi$ be such that in one step we come to this (gauge equivalent) equation:

$$
\begin{array}{r}
\widetilde{u}(x, t)=u-2(\log \varphi)_{x x}=u-C(t), \\
i \varphi_{t}=-\varphi_{x x}+u(x, t) \varphi .
\end{array}
$$

We obtain the relation

$$
\varphi(x, t)=\exp \left(\frac{C x^{2}}{2}+A x+B\right),
$$


where $A, B, C$ are arbitrary functions of $t$. For $C \neq 0$ we obtain an equation of the form

$$
\varphi(x, t)=\exp \left(\frac{C\left(x-x_{0}(t)\right)^{2}}{2}+D(t)\right) .
$$

For $C \equiv 0$ we have $(A \neq 0)$ :

$$
\varphi(x, t)=\exp (A(t) x+B)=\exp \left(A(t)\left(x-x_{0}(t)\right)\right) .
$$

It is easy to prove the following lemma.

Lemma 3 The Darboux chain for a non-stationary Schrödinger equation is cyclic of period 1 if and only if the potential $u(x, t)$ has the form

$$
u(x, t)=(\alpha(t) x)^{2}+\beta(t) x+\gamma(t),
$$

and the chosen solution $\varphi(x, t)$ is given in the form (19), where the equalities

$$
\begin{aligned}
& i \dot{C}=2\left(\alpha^{2}-C^{2}\right), \\
& i \dot{A}=\beta-2 A C, \\
& i \dot{B}=\gamma-\left(A^{2}+C\right)
\end{aligned}
$$

are satisfied. The dynamics of $x_{0}$ from (20) is as follows:

$$
\dot{x}_{0}=\frac{2 \alpha^{2} x_{0}-\beta}{C} .
$$

The proof is obtained by simple calculation.

We consider two cases.

Case 1. Let $w+i v=C \neq 0$. Then either $\alpha^{2} \neq 0$ or $\alpha^{2} \equiv 0$. In the case $\alpha \neq 0$ we have a 'moving oscillator of variable form'.

Lemma 4 Solutions of the form (19), where $w\left(t_{0}\right)<0$, are in $\mathcal{L}_{2}(\mathbb{R})$ with respect to the variable $x$ for any $t \geq t_{0}$ if the condition

$$
\operatorname{Im} \alpha^{2}(t) \leq 0
$$

is satisfied.

Proof The domain $\operatorname{Re} C<0$ is invariant for the system (21), since for $C=$ $w+i v$ we have $\dot{w} \leq 0$ for $w=0$ by virtue of the equation

$$
\dot{w}=2\left(\operatorname{Im} \alpha^{2}-2 v w\right) .
$$

We have proved Lemma 4.

Let $\alpha^{2}=$ const $>0$. Then the condition $C=-\alpha, \alpha \in \mathbb{R}$, gives a stationary solution for $C$ in the domain $u=\operatorname{Re} C<0$. All trajectories in this domain are 
periodic and the equation is easily integrated. The trajectories are given by the equation $H(w, v)=$ const, where $H=\left((\alpha-w)^{2}+v^{2}\right) / w$.

Remark 1 A set of operators of the form $Q_{a}=\partial_{x}+a x$ satisfies the commutation relations

$$
\left[Q_{a}, Q_{b}\right]=(a-b) \cdot 1, \quad Q^{+}=-Q_{-\bar{a}}
$$

for all complex $a, b$. The commutators of all these operators with $H_{\alpha}=Q_{\alpha} Q_{\alpha}^{+}$, where $Q_{\alpha}^{+}=-Q_{-\bar{\alpha}}$, have the form

$$
\left[Q_{a}, H_{\alpha}\right]=(a-\alpha) Q_{-\bar{\alpha}}+(a+\bar{\alpha}) Q_{\alpha}
$$

The usual coherent states are eigenvectors of the annihilation operators. The states we have studied are eigenvectors of the operators

$$
Q_{-C} \psi=\gamma \psi, \quad \operatorname{Re} C<0
$$

Here $\gamma \in \mathbb{C}$, and in formula (20) above $\gamma=C x_{0}$. Hence, the states (20) are determined purely algebraically by means of the Lie algebra (23), which contains the oscillator Hamiltonian $H=H_{\alpha}$.

Remark 2 The well-known 'coherent states' of the oscillator are eigenvectors of the annihilation operator

$$
\begin{array}{r}
Q \psi_{\gamma}=\gamma \psi_{\gamma}, \quad \gamma \in \mathbb{C} \\
Q=\partial_{x}+\alpha x, \quad \psi_{\gamma}(x)=e^{-\alpha(x-\gamma / \alpha)^{2} / 2} .
\end{array}
$$

Using these functions as the initial conditions, we obtain solutions of the Schrödinger equation in the particular form (20), where $C=-\alpha=$ const $<0$. For $x_{0}(t)$ we obtain motion along the imaginary straight line:

$$
\begin{array}{r}
x_{0}(t)=x_{0}(0)+2 i t \\
\psi_{\gamma}(x, t)=e^{-\alpha\left(x-\gamma / \alpha-x_{0}(t)\right)^{2} / 2+B(t)}
\end{array}
$$

Question For $t=0$ suppose we have $C(0)=q$ for all solutions of (20). For which $\left(x_{0, q}, q\right)$ is the set of functions $\varphi_{q}(x, 0)$, where $\varphi_{q}(x, 0)=e^{q\left(x-x_{0, q}\right)^{2} / 2}$, complete in $\mathcal{L}_{2}(\mathbb{R})$, where $q$ runs along the curve from the point $q_{0}=-\alpha$ to $q=\infty$ in such a way that $\operatorname{Re} q<0$ ? How can we choose a minimal complete basis if this set is overfull? Naturally we can choose a curve $\infty<q \leq-\alpha$ and translations $x_{0, q}$ such that the set will be complete, $q \in \mathbb{R}$.

Remark 3 We also note that for the oscillator $u=\alpha^{2} x^{2}, \alpha^{2}=$ const $>0$, we can perform the Darboux transformation, starting from the second solution in $\mathcal{L}_{2}(\mathbb{R})$ :

$$
\left.\varphi(x, t)\right|_{t=0}=P_{n}(x) e^{-\alpha x^{2} / 2}, \quad \alpha>0
$$


where $P_{n}(x)$ is an arbitrary polynomial in $x$ of degree $n$ (here $\alpha=C=$ const). For the potential $\widetilde{u}(x, t)$ we have

$$
\widetilde{u}=\alpha^{2} x^{2}-2(\log \varphi)_{x x}=\alpha^{2} x^{2}-\sum_{j} \frac{2}{\left(x-x_{j}(t)\right)^{2}} .
$$

We determine the dynamics of $\varphi(x, t)$ by expanding $P_{n}(x)$ in terms of the Hermite polynomials, that is, in terms of eigenfunctions of the oscillator. There arises a motion of the poles of the potential $\widetilde{u}(x, t)$ with respect to $t$ (or the motion with respect to $t$ of the polynomial $\left.P_{n}(x, t), P_{n}(x, 0)=P_{n}(x)\right)$ such that

$$
\begin{array}{r}
P_{n}(x, t)=\sum e^{-i \lambda_{j} t} a_{j} H_{j}(x) \\
P_{n}(x)=\sum_{j \leq n} a_{j} H_{j}, \quad \lambda_{j}=\alpha+2 j \alpha .
\end{array}
$$

$H_{j}(x)$ are the Hermite polynomials defined by the formulae

$$
H_{0}=1, \quad H_{j}(x)=\left(\left(Q^{+}\right)^{j} e^{-\alpha x^{2}}\right) / e^{-\alpha x^{2}},
$$

where $Q^{+}=\partial_{x}-\alpha x$. We have

$$
P_{n}(x, t)=a_{n} e^{-i(2 n+1) \alpha t} \prod_{j=1}^{n}\left(x-x_{j}(t)\right) .
$$

We can obtain a more extensive family of solutions of this type from initial conditions of the form

$$
\varphi(x, 0)=P_{n}(x) e^{C(0)\left(x-x_{0}\right)^{2} / 2},
$$

applying the operators $Q_{-C}^{+}=Q_{\bar{C}}$ to the initial conditions for solutions of (20). The dynamics of their poles can also be of interest.

Case 2. Let $C \equiv 0$. Then $\alpha \equiv 0$ and we have

$$
u(x, t)=\beta x+\gamma, \quad \varphi(x, t)=\exp \left(i A(t)\left(x-x_{0}(t)\right)\right) .
$$

This is a physically reasonable case when $\beta=E(t)$ and is periodic in $t$. In this case (known as the integrable case of L. V. Keldysh) we can also find a general solution.

We note with interest that even in the stationary case $u(x)=\beta x+\gamma$ when $\beta, \gamma$ are constants, it is more convenient to use the basis of non-stationary solutions $\varphi(x, t)$, which have the form of plane waves for fixed time.

Ultimately only the non-stationary Schrödinger equation is a law of nature. If the force $u_{x}$ does not depend on time, then we can consider the Hamiltonian $\widehat{H}$ in an appropriate gauge as stationary. As usual, in this case in place of the non-stationary equation

$$
i \psi_{t}=\widehat{H} \psi
$$


we can use the Fourier method and solve the stationary problem

$$
\widehat{H} \psi=\lambda \psi
$$

in the space $\mathcal{L}_{2}(\mathbb{R})$.

After this, we shall have the general solution in the form of finite or continuous linear combinations

$$
\psi(x, t)=\sum_{j} a_{j} \psi_{j} e^{-i \lambda_{j} t}, \quad \widehat{H} \psi_{j}=\lambda_{j} \psi_{j} .
$$

However, this generally accepted approach makes sense only if the spectral problem is sufficiently well solved: it is only a mathematical trick, nothing more.

If the stationary problem is not sufficiently well solved, then the Fourier method is not appropriate. An example of such a situation is the constant electric field $u(x)=E x$, where the basis of non-stationary solutions (plane waves) is much simpler, as we mentioned above, than the eigenfunctions of the stationary operator $\widehat{H}$ (Airey functions).

The advantage of this approach can be particularly important in the case when the potential has the form

$$
u(x)=E x+u_{0}(x),
$$

where the function $u_{0}(x)$ is periodic (a constant electric field is applied to the crystal). Already in 15 the perspectives of the non-stationary approach in this case were discussed. A physically reasonable general non-stationary case is

$$
u(x, t)=E(x, t),
$$

where $E(x, t)$ is the electric field, periodic in both variables. If the potential $u(x, t)$ is doubly periodic ('zero electric current'), then we have an extensive family of algebro-geometric exactly soluble equations, where $u(x, t)$ is expressed in terms of theta-functions of Riemann surfaces (see [16], [17]). We construct the Bloch solution $\psi$ of $(10)$ :

$$
\begin{aligned}
& \psi\left(x+T_{1}, t\right)=e^{i p_{1} T_{1}} \psi(x, t), \\
& \psi\left(x, t+T_{0}\right)=e^{i p_{0} T_{0}} \psi(x, t),
\end{aligned}
$$

as the 'Baker-Akhiezer function' on some Riemann surface $\Gamma$ of finite genus, that is, the parameters $p_{1}, p_{0}$ are connected by the equation of the algebraic curve $\Gamma$. These potentials were used for solutions of the 'KP equation', where $t$ is renamed as $y$, and time, denoted by $t$, is the third variable. Undoubtedly these solutions of Krichever [16], [17] are obtained from the cyclic chains of Darboux transformations, but a rigorous assertion has not been proved up to now.

More complex is the case of a non-zero 'electric flux'. The case $E(t)=\beta$ is well known as the integrable case of Keldysh.

In these cases, to understand the problem it would be useful to broaden the number of known exact solutions. In 15 under certain conditions ('quantization of the electric flux' using an elementary cell in the $(x, t)$ plane) Novikov 
introduced electric Bloch functions, which are eigenfunctions for 'electric translations' by periods, but their analytical properties for $E \neq 0$ have not been understood and formulated up to now.

We transform the Schrödinger equation (10) using the gauge transformation

$$
\psi^{\prime}=e^{i F(x, t)} \psi, \quad L^{\prime}=e^{i F} L e^{-i F},
$$

so that

$$
i \psi_{t}^{\prime}=-\left(\partial_{x}+i A_{1}(x, t)\right)^{2} \psi^{\prime} .
$$

For this we need to take $F_{t}=u(x, t), A_{1}=-F_{x}$.

Let $u=\beta(t) x$. We have $F=A(t), \dot{A}=\beta(t)$. We have a solution of form

$$
\psi_{k}^{\prime}=e^{i k x} \varphi_{k}(t)
$$

where

$$
i \dot{\varphi}_{k}=(k-A(t))^{2} \varphi_{k}, \quad \varphi_{k}=e^{-i \int^{t}(k-A(\tau))^{2}} d \tau .
$$

For

$$
\beta(t)=E=\mathrm{const}
$$

we have

$$
\psi_{k}^{\prime}=e^{i k x} e^{i E^{2} / 3}\left(k E^{-1}-t\right)^{3} .
$$

This is a basis in the space of solutions. tion,

The operators of 'electric translations', which preserve the Schrödinger equa-

$$
i \nabla_{t} \psi=-\nabla_{x}^{2} \psi
$$

where $\nabla_{t}=\partial_{t}+i A_{0}, \nabla_{x}=\partial_{x}+i A_{1}$, are fully analogous to magnetic translations. For a periodic force $E(x, t)$,

$$
E(x, t)=A_{0 x}-A_{1 t}=E\left(x+T_{1}, t\right)=E\left(x, t+T_{0}\right),
$$

the electric translations that commute with (31) are given by

$$
\widehat{T}_{1} \psi=e^{i F_{0}(x, t)} \psi\left(x+T_{1}, t\right), \quad \widehat{T}_{0} \psi=e^{i F_{1}(x, t)} \psi\left(x, t+T_{0}\right),
$$

where $d F_{0}=A\left(t+T_{0}\right)-A(t), d F_{1}=A\left(x+T_{1}\right)-A(x), A=A_{0} d t+A_{1} d x$. We have the identity

$$
\widehat{T}_{1} \widehat{T}_{0}-\widehat{T}_{0} \widehat{T}_{1}=e^{i \Phi_{01}}, \quad \Phi_{01}=\int_{0}^{T_{0}} \int_{0}^{T_{1}} E(x, t) d x d t .
$$

If $\Phi_{01}=2 \pi q, q \in \mathbb{Z}$, then the group of 'electric translations' is commutative (as in the magnetic case). In the particular case (29) we obtain

$$
\widehat{T}_{1} \psi_{k}^{\prime}=T_{1} \psi_{k}^{\prime}=e^{i k T_{1}} \psi_{k}^{\prime}, \quad \widehat{T}_{0} \psi_{k}^{\prime}=\psi_{k-E T_{0}}^{\prime} .
$$


The electric Bloch states, which are eigenstates for $\widehat{T}_{1}, \widehat{T}_{0}$ under the condition $E T_{0} T_{1}=2 \pi q, q \in \mathbb{Z}$, are formally written in the form

$$
\psi\left(x, t, p_{0}, p_{1}\right)=\sum_{m \in \mathbb{Z}} e^{-i m p_{0} T_{0}} \widehat{T}_{0}^{m}\left(\psi_{k}^{\prime}(x, t)\right) .
$$

Obviously we have

$$
\begin{array}{r}
\widehat{T}_{0} \psi=e^{i p_{0} T_{0}} \psi, \\
\widehat{T}_{1} \psi=e^{i p_{1} T_{1}} \psi, \quad p_{1}=k \quad\left(\bmod E T_{0}\right) .
\end{array}
$$

These are complicated generalized functions. For $\beta(t)=E$ we have, for example,

$$
\psi\left(x, t, p_{0}, p_{1}\right)=e^{i k x}\left(\sum_{m \in \mathbb{Z}} e^{i\left(-m T_{0}\left(p_{0}+E x\right)+\frac{E^{2}}{3}\left(k E^{-1}-t-m T_{0}\right)^{3}\right)}\right) .
$$

(As opposed to the magnetic case, where the functions $\varphi_{k}$ rapidly decrease as $|x| \rightarrow \infty$, and the analogue of the series (35) converges, giving analytic functions.)

As the simplest example we show that under the 'strong integer' condition $q, s, l \in \mathbb{Z}$

$$
E T_{0} T_{1}=2 \pi q, \quad E^{2} / 3=2 \pi s, \quad T_{0}=l,
$$

we obtain for $t_{0}=k E^{-1}, 0 \leq k \leq E T_{0}$

$$
\frac{1}{2 \pi} \psi\left(x, t_{0}, p_{0}, p_{1}\right)=e^{i k x} \sum_{m \in \mathbb{Z}} \delta\left(T_{0}\left(p_{0}+E x\right)+2 \pi m\right), \quad k=p_{1} \quad\left(\bmod E T_{0}\right) .
$$

Hence, under the condition (37) the electric Bloch states are obtained by solving the Cauchy problem with singular initial condition (38). In the survey [15], although the scheme and the ideology were correct, misprints and errors were made in the formulae of the text in the description of the electric Bloch states.

The function (36) is a sum of two functions $\psi=\psi_{+}+\psi_{-}$, where $\psi_{+}$is the boundary value of the function which is analytic for $\operatorname{Im} p_{0}>0$, and $\psi_{-}$is the boundary value of the function which is analytic for $\operatorname{Im} p_{0}<0$. This property is also possibly true in the general case for electric Bloch states. Our series (36) converges for $p_{1}=k_{\mathbb{R}}+i \kappa^{2}, \kappa^{2}>0$, as we can easily see from (36), so in the variable $k=p_{1}\left(\bmod E T_{0}\right)$ the function $(36)$ is the boundary value of an analytic function.

In the case of a constant field under the strong integer condition (37), starting from (38) the electric Bloch function can be completely calculated. We recall that a theta-function with zero characteristics has the form

$$
\theta_{\tau}(z)=\sum_{m \in \mathbb{Z}} e^{i \tau m^{2} / 2+m z}
$$

and is well defined for $\operatorname{Im} \tau>0$. 
Comparing with (36) we have finally

$\psi\left(x, t, p_{0}, p_{1}\right)=\theta_{\tau}(z), \quad \tau=2\left(E T_{0}\right)^{2}\left(\frac{k}{E}-t\right)^{2}+i 0, \quad z=i\left(E T_{0}\right)\left(k-E t-\frac{p_{0}}{E}\right)$,

that is, the limit of the theta function when $\operatorname{Im} \tau \rightarrow+0$,

$$
\begin{array}{r}
p_{1}=k \quad\left(\bmod E T_{0}\right), \\
\widehat{T}_{1} \psi=e^{i p_{0} T_{0}} \psi, \quad \widehat{T}_{2} \psi=e^{i p_{1} T_{1}} \psi, \\
T_{0} \in \mathbb{Z}, \quad E^{2} / 3 \in 2 \pi \mathbb{Z}, \quad E T_{0} T_{1} \in 2 \pi \mathbb{Z} .
\end{array}
$$

In the remaining cases there arise generalized functions (distributions) given by infinite trigonometric sums that are cubic in $m \in \mathbb{Z}$. Apparently, quantities of this type have not been studied.

Remark 4 In the case $\alpha=0$ and $\beta=$ const $=E$ we have a special family of solutions such that $C \neq 0$ for $t \in \mathbb{R}$. From (21) we find that $i /(2 t+i p)=C$, $p \in \mathbb{R}$,

$$
\varphi(x, t)=\exp \left\{i \frac{\left(x-x_{0}(t)\right)^{2}}{4 t+2 i p}+D(t)\right\} .
$$

We have

$$
-x_{0}(0)+x_{0}(t)=\beta t^{2}
$$

where $p<0$.

The motion of the centre $x_{0}$ for $p=0$ is the motion of a classical particle in the constant field $E=\beta$. In this solution we have coincidence of the classical and quantum pictures.

We have presented these elementary solutions here to demonstrate on the example $N=1$ how the problem on cyclic Darboux chains for the one-dimensional non-stationary Schrödinger operator should be correctly formulated.

\section{Definition 2}

We call the transformation

$$
u \rightarrow u+\gamma(t), \quad \psi \rightarrow e^{-i g(t)}
$$

the gauge transformation of a one-dimensional non-stationary Schrödinger operator, where $\gamma(t)$ is an arbitrary function of time, and $\dot{g}=\gamma$.

Definition 3 The chain of Darboux transformations for a one-dimensional nonstationary Schrödinger operator is a sequence of equations

$$
i \psi_{t}=L_{j} \psi=-\psi_{x x}+u_{j}(x, t) \psi
$$

such that the operator $L_{j+1}$ is obtained from $L_{j}$ by the Darboux transformation and then by the gauge transformation acting on the function $\gamma_{j}(t)$ :

$$
\begin{gathered}
u_{j+1}(x, t)=\widetilde{u}_{j}(x, t)+\gamma_{j}(t), \\
\widetilde{u}_{i}=u_{i}-2\left(\log \varphi_{i}\right)_{x x}, \\
i \varphi_{j t}=-\varphi_{j x x}+u_{j}(x, t) \varphi .
\end{gathered}
$$


Hence, the Darboux chain is determined by a choice of solutions $\varphi_{j}$ and arbitrary functions of time $\gamma_{j}(t), j \in \mathbb{Z}$. In the case of a cyclic chain of period $N$ we have $N$ solutions $\varphi_{j}$ and $N$ arbitrary functions $\gamma_{j}(t)$.

\section{$\S 3$. One-dimensional difference operators}

We consider a difference operator $L$ of the second order

$$
L \psi_{n}=c_{n-1} \psi_{n-1}+v_{n} \psi_{n}+c_{n} \psi_{n+1} .
$$

¿From the theory of solitons we know [3], [18]- [20], that for the conservation, in the discrete case, of the latent algebraic symmetries of Schrödinger operators (such as isospectral deformations of KdV type and the Bäcklund-DarbouxEuler transformation) it is necessary to introduce the covariant translations $\exp \left(\nabla_{x}\right)$ in place of the translations $T=\exp \left(\partial_{x}\right)$, where $\exp \left(\nabla_{x}\right)=c_{n} T \psi_{n} \rightarrow$ $\psi_{n+1} c_{n}$ (the 'covariant translation').

After this, the theory of the corresponding class of difference operators (43) is similar to the continuous case: there arise isospectral deformations of the type of the Toda chain for (43) or of the 'discrete KdV' for (43) under the condition $v_{n}=0$. There also arise analogues of Darboux transformations starting from factorization:

a) $\alpha+L=Q Q^{+}, T^{+}=T^{-1}, Q=a_{n}+b_{n} T, Q^{+}=a_{n}+T^{-1} b_{n}$;

b) $\alpha+L=\widehat{Q}^{+} \widehat{Q}, \widehat{Q}=\widehat{a}_{n}+\widehat{b}_{n} T$.

For the factorization, as before, we have to solve a difference analogue of the Riccati equation

$$
\begin{aligned}
& v_{n}+\alpha=a_{n}^{2}+b_{n}^{2}, \quad a_{n+1} b_{n}=c_{n}, \\
& v_{n}+\alpha=\widehat{a}_{n}^{2}+\widehat{b}_{n-1}^{2}, \quad c_{n}=\widehat{a}_{n} \widehat{b}_{n} .
\end{aligned}
$$

Factorization is also possible in the general non-self-adjoint case (direct and inverse):

$L=p_{n} T^{-1}+q_{n}+r_{n} T=\left(a_{n}+b_{n} T\right)\left(x_{n}+y_{n} T^{-1}\right)=\left(c_{n}+d_{n} T^{-1}\right)\left(v_{n}+w_{n} T\right)$.

The Bäcklund-Darboux transformations are defined as in the continuous case $(21],[22])$ :

$$
\begin{gathered}
B_{\alpha}=B T_{\alpha}, \quad B_{\alpha}^{\prime}=B^{\prime} T_{\alpha}, \\
B L \longmapsto \widetilde{L}=Q^{+} Q, \quad \psi \longmapsto \widetilde{\psi}=Q^{+} \psi, \\
B^{\prime} L \longmapsto \widehat{L}^{\prime}=\widehat{Q} \widehat{Q}^{+}, \quad \psi \longmapsto \widehat{\psi}^{\prime}=\widehat{Q} \psi, \\
T_{\alpha} L \longmapsto L+\alpha,
\end{gathered}
$$


$L=Q Q^{+}$(in all cases we first perform factorization and then permute the non-commutative factors, that is, the first-order operators).

There naturally arises the problem on cyclic chains ([21], [22]), for example, on the direct transformations

$$
B_{\alpha_{N}} \circ B_{\alpha_{N-1}} \circ \cdots \circ B_{\alpha_{0}}(L)=L .
$$

It was established in [22] that if $\sum \alpha_{j}=0$, then $L$ is a finite-zone operator with Riemann surface ('spectrum') of genus $g \leq[N / 2]$. If the $\sum \alpha_{j}=\alpha \neq 0$, then we arrive at the difference analogues of the theory [12], [13].

We should take into account that the transformations $B_{\alpha}$ and $B_{\alpha}^{\prime}$ depend also on a continuous parameter, that is, on the choice of a solution of the Riccati difference equation (44) or (45), which depends on the choice of the initial point $b_{0}\left(\right.$ or $\left.\widehat{b}_{0}\right)$ and the set of signs $\left(\operatorname{sgn} a_{n}\right)$. We denote this joint parameter by $\kappa$. The problem of an appropriate choice of signs is discussed, for example, in 21]. In any case, we have the relation

$$
B^{\prime \kappa^{\prime}} B^{\kappa} L=L
$$

for any $L$, where the choice of the parameter $\kappa^{\prime}$ is naturally consistent with $\kappa$.

In principle we can consider general words in the group generated by the transformations $T_{\alpha^{\prime}} B^{\prime \kappa}, B^{\kappa}$ for all $(\kappa, \alpha)$ and formulate the cyclicity condition with respect to them. Hence, we have the following conclusion.

Conclusion. The set of Darboux transformations for a one-dimensional difference operator is larger than for a continuous one because of the two different types of factorizations (above). In 21-223 the cyclicity condition was studied for 'direct' chains of the form (49) only.

It is interesting to look at the case $N=1$, where we obtain an analogue of the oscillator, starting from the Heisenberg relation:

$$
Q Q^{+}=Q^{+} Q+\alpha
$$

This equation has a solution

$$
Q^{+}=1+\sqrt{a+b n} T .
$$

The coefficients of such an operator $Q$ cannot be real for all $n$.

Lemma 5 We assume that the 'quantization condition'

$$
a+b n=b\left(n_{0}+n\right)
$$

is satisfied, where $n_{0}=a / b \in \mathbb{Z}, a, b \in \mathbb{R}, b>0$.

Then the operators $Q$ and $Q^{+}$are well defined in the subspace of functions $\psi$ such that

$$
\psi_{n}=0, \quad n+n_{0} \leq 0, \quad \psi \in \mathcal{L}_{2}(\mathbb{R}) .
$$


This lemma is easily proved by direct verification.

As was shown in [22], the ground state is defined according to Dirac:

$$
Q^{+} \psi_{0}=0 .
$$

At the same time we have 22

$$
\psi_{0 n}^{2}=1 /\left(\alpha^{k-1}(k-1) !\right), \quad k=n+n_{0}>0 .
$$

Hence, $\psi_{0 n}^{2}$ is the Poisson distribution. Applying creation operators we obtain the eigenfunctions

$$
Q^{k} \psi_{0}=P_{k}\left(n+n_{0}\right) \psi_{0 n},
$$

where $P_{k}(n)$ are the well-known Charlier polynomials, orthogonal with respect to the weight $\left(\psi_{0 n}^{2}\right)$, that is, the Poisson distribution on $\mathbb{Z}_{+}$.

Hence, in the difference case the Heisenberg relation (50) is realized (among first-order operators) on the positive half-line $\mathbb{Z}_{+}$.

There is also another analogue, the ' $q$-analogue' of the oscillator, where the operators $Q, Q^{+}$depend on two parameters:

$$
Q_{c, a}=1+c a^{n} T .
$$

Lemma 6 We have the relation

$$
Q_{c, a} Q_{c, a}^{+}-1=a^{2}\left(Q_{c_{1}, a}^{+} Q_{c_{1}, a}-1\right), \quad c_{1}=c a^{2} .
$$

The proof consists of direct verification.

In 22] the following theorem was proved (parts of it were already given in 210 ).

Theorem 1 1) The transformation

$$
\tau n \rightarrow 1-n
$$

acts on the operators $Q_{c}, Q_{c}^{+}$in the following way:

$$
\tau Q_{c, a}=Q_{c, a^{-1}}^{+} \tau \text {. }
$$

2) The equation $Q_{c} \psi_{0}=0$ has a solution in $\mathcal{L}_{2}(\mathbb{Z})$ under the condition $|a|>$ 1. The equation $Q_{c}^{+} \psi_{0}=0$ has a solution in $\mathcal{L}_{2}(\mathbb{Z})$ under the condition $|a|<1$.

3) The spectrum of the operator $L_{c, a}=Q_{c, a} Q_{c, a}^{+}$in the interval $0 \leq \lambda<1$ has the form

$$
\begin{array}{llll}
\lambda_{n}=1-a^{-2 n}, & n \geq 0, & \text { if } & |a|>1, \\
\lambda_{n}=1-a^{2 n}, & n \geq 1, & \text { if } & |a|<1 .
\end{array}
$$

Analogously, the spectrum of the operator $\widetilde{L}_{c, a}=Q_{c, a}^{+} Q_{c, a}$ in the interval $0 \leq$ $\lambda<1$ is given by

$$
\begin{array}{llll}
\lambda_{n}=1-a^{-2 n}, & n \geq 1, \quad \text { if } & |a|>1, \\
\lambda_{n}=1-a^{2 n}, & n \geq 0, & \text { if } & |a|<1 .
\end{array}
$$


Problem Investigate the spectrum of the operators $L=Q Q^{+}, \widetilde{L}=Q^{+} Q$ in $\mathcal{L}_{2}(\mathbb{Z})$ in the domain $\lambda \geq 1$, if $Q$ has the form (54). According to the hypothesis [22], this spectrum is continuous and Lebesgue (see $\S 6$ below).

\section{$\S 4$. The Laplace transformation and the two- dimensional Schrödinger operator in a magnetic field}

We write a general $n$-dimensional stationary scalar Schrödinger operator in Euclidean space in the form

$$
L=-\frac{1}{2} \sum\left(\partial_{\alpha}+i A_{\alpha}(x)\right)^{2}+U(x),
$$

where $x=\left(x^{1}, \ldots, x^{n}\right), \partial_{\alpha}=\partial / \partial x^{\alpha}$. In particular, the 2-form of the 'magnetic field' has the form

$$
\begin{gathered}
H=\sum_{\alpha<\beta} H_{\alpha \beta} d x^{\alpha} \wedge d x^{\beta}, \quad d H=0, \\
H_{\alpha \beta}=\partial_{\alpha} A_{\beta}-\partial_{\beta} A_{\alpha}=-H_{\alpha \beta} .
\end{gathered}
$$

The electric force has the form

$$
\vec{E}=\left(\partial_{1} U, \ldots, \partial_{n} U\right),
$$

that is, the potential $U$ is defined up to a constant. By the gauge transformations

$$
L \longmapsto e^{f} L e^{-f}, \quad \psi \longmapsto e^{f} \psi
$$

we can change the 'vector-potential':

$$
A_{\alpha} \longmapsto A_{\alpha}-i \partial_{\alpha} f
$$

In the physically comprehensible self-adjoint case we have a real magnetic field and the potential: $H_{\alpha \beta} \in \mathbb{R}, U \in \mathbb{R}$, and we choose a real vector-potential $A_{\alpha} \in \mathbb{R}$. Then the gauge transformations have the form (62) (in the class of stationary operators), where $f=i \varphi(x), \varphi(x) \in \mathbb{R}$. The operator obtained by this transformation possesses as before the formal self-adjoint property with respect to the standard scalar product in the Hilbert space $\mathcal{L}_{2}(\mathbb{R})$ :

$$
\left\langle\psi_{1}, \psi_{2}\right\rangle=\int_{\mathbb{R}^{n}} \psi_{1} \bar{\psi}_{2} d^{n} x
$$

An important class consists of operators with smooth real magnetic and electric fields, which are periodic with respect to some lattice $\Gamma \subset \mathbb{R}^{n}$ of rank $n$. In this case we introduce the following notions. 
Definition 4 We say that an operator $L$ is topologically non-trivial if the magnetic field (60) is the non-zero cohomology class of a torus,

$$
0 \neq[H] \in H^{2}\left(\mathbb{T}^{n}, \mathbb{R}\right) .
$$

Definition 5 We say that an operator $L$ possesses a topologically non-trivial electric field if the 1-form of the electric field (in the non-relativistic stationary formalism) is the non-zero cohomology class of a torus:

$$
0 \neq[E] \in H^{1}\left(\mathbb{T}^{n}, \mathbb{R}\right),
$$

$E=E_{\alpha} d x^{\alpha}, E_{\alpha}=\partial_{\alpha} U(x)$. In other words, the potential has the form

$$
U(x)=E_{0 \alpha} x^{\alpha}+U_{0}(x),
$$

where the function $U_{0}(x)$ is periodic with respect to the lattice, $E_{0}=$ const $\epsilon$ $\left(\mathbb{R}^{n} \backslash 0\right)$.

A topologically non-trivial electric field has arisen already in the one-dimensional case. As we showed in $\S 2$, it was appropriate to study such a situation in the framework of the non-stationary $(x, t)$-formalism, even if the electric force is stationary.

For magnetic fields we use the stationary formalism. If $\gamma_{1}, \ldots, \gamma_{n} \in \Gamma$ is the basis of the lattice, then $H_{\alpha \beta}\left(x+\vec{\gamma}_{j}\right)=H_{\alpha \beta}(x)$. For the vector-potential we have

$$
A_{\alpha}\left(x+\vec{\gamma}_{j}\right)=A_{\alpha}(x)+\partial_{\alpha} \varphi_{j}(x),
$$

where $\varphi_{j}$ is some function of $x=\left(x^{1}, \ldots, x^{n}\right)$.

We define 'magnetic translations' (15), which have been well known in the physical literature since the mid-60s:

$$
\widehat{T}_{j} \psi(x)=\psi\left(x+\vec{\gamma}_{j}\right) e^{i \varphi_{j}(x)} .
$$

We have the relation

$$
\widehat{T}_{j} \widehat{T}_{k}=e^{i \Phi_{j k}} \widehat{T}_{k} \widehat{T}_{j},
$$

where $\Phi_{j k}$ is the flux of a magnetic field through an elementary two-dimensional cell of the lattice in the plane $\left(\vec{\gamma}_{j}, \vec{\gamma}_{k}\right)$, or the scalar product (integral) of the cocycle $[H]$ with the basis 2 -cycle $Z_{j k} \in H_{2}\left(\mathbb{T}^{n}, \mathbb{Z}\right)$.

In the two-dimensional case $n=2$ we can present the operator $L$ in a factorized form, which we write as

$$
2 L=(\bar{\partial}+B)(\partial+A)+2 W,
$$

where $A(z, \bar{z}), B(z, \bar{z}), W(z, \bar{z})$ are some functions of $z, \bar{z}$,

$$
z=x+i y, \quad \partial=\partial_{x}-i \partial_{y} .
$$


The magnetic field has the form

$$
2 H=B_{z}-A_{\bar{z}} .
$$

If $H, W$ are real, then we can, by using the gauge transformation, bring the operator to the self-adjoint form, so we have

$$
\begin{array}{r}
2 L=Q_{1} Q_{2}+2 W, \\
Q_{1}=\bar{\partial}+B, \quad Q_{2}=\partial+A=-Q_{1}^{+}, \quad A=-\bar{B} .
\end{array}
$$

The operator $L$ is always written in the form (70), that is, it 'admits factorization' in our terminology.

The operator $L$ also admits an opposite factorization of the form

$$
2 L=Q_{2} Q_{1}+2 V,
$$

where $V=W+H, Q_{2}=\partial+A, Q_{1}=\bar{\partial}+B$.

Definition 6 The Laplace transformation is the transformation

$$
L \longmapsto \widetilde{L}, \quad 2 \widetilde{L}=W Q_{2} W^{-1} Q_{1}+2 W,
$$

where $2 L=Q_{1} Q_{2}+2 W$.

Lemma 7 a) If $\psi$ is an arbitrary solution of the equation $L \psi=0$, then the function $\widetilde{\psi}=Q_{2} \psi$ satisfies the equation $\widetilde{L} \widetilde{\psi}=0$.

b) If $W=W_{0}=$ const, then for any solution $\psi$ of the equation $L \psi=\lambda \psi$ the function $\widetilde{\psi}=Q_{2} \psi$ satisfies the equation $\widetilde{L} \widetilde{\psi}=\lambda \widetilde{\psi}$. In this case $2 \widetilde{L}=$ $Q_{2} Q_{1}+2 W_{0}$.

Proof The proof of Lemma 7 is very simple. Since $L \psi=0$, we have

$$
-2 W \psi=Q_{1} Q_{2} \psi=Q_{1} \widetilde{\psi} .
$$

¿From this it follows that

$$
\begin{array}{r}
-2 \psi=W^{-1} Q_{1} \widetilde{\psi}, \\
-2 \widetilde{\psi}=-2 Q_{2} \psi=Q_{2} W^{-1} Q_{1} \widetilde{\psi}, \\
-2 W \widetilde{\psi}=W Q_{2} W^{-1} Q_{1} \widetilde{\psi} .
\end{array}
$$

The lemma has been proved for the case a). In the case b), for $W=W_{0}=$ const, the proof is exactly the same.

Lemma 8 The Laplace transformation for the equation $L \psi=0$ commutes with the gauge transformation (62). If $W=W_{0}=$ const, then this applies to the whole family of equations $L \psi=\lambda \psi$.

In particular, this transformation is defined on the gauge invariant quantities $H, W$ :

$$
\widetilde{H}=H+\frac{1}{2} \partial \bar{\partial} \log W, \quad \widetilde{W}=W+\widetilde{H} .
$$


The proof of this lemma is obtained by direct verification. We omit it.

Remark 5 The classicists, beginning with Laplace, considered this transformation for the hyperbolic equation

1) $2 L=\left(\partial_{x}+A\right)\left(\partial_{y}+B\right)+2 W$,

$$
2 \widetilde{L}=W\left(\partial_{y}+B\right) W^{-1}\left(\partial_{x}+A\right)+2 W ;
$$

2) $2 L=\left(\partial_{y}+B\right)\left(\partial_{x}+A\right)+2 V, \quad V=W+H$,

$$
2 \widetilde{L}^{\prime}=V\left(\partial_{x}+A\right) V^{-1}\left(\partial_{y}+B\right)+2 V .
$$

Here the pair of mutually inverse Laplace transformations acts on solutions of the equation $L \psi=0$ :

1) $L \longmapsto \widetilde{L}, \quad \psi \longmapsto \widetilde{\psi}=\left(\partial_{y}+B\right) \psi$;

2) $L \longmapsto \widetilde{L}^{\prime}, \quad \psi \longmapsto \widetilde{\psi}^{\prime}=\left(\partial_{x}+A\right) \psi$.

As before, the gauge invariants have the form $H, W$ or $H, V$, where

$$
2 H=\partial_{x} B-\partial_{y} A .
$$

For these invariants the Laplace transformations have the form analogous to (73):

$$
\begin{aligned}
& \text { 1) } \widetilde{H}=H+\frac{1}{2} \partial_{1} \partial_{2} \log W, \quad \widetilde{W}=W+\widetilde{H} \text {; } \\
& \text { 2) } \widetilde{H}^{\prime}=H+\frac{1}{2} \partial_{1} \partial_{2} \log V, \quad \widetilde{V}^{\prime}=V+\widetilde{H}^{\prime} \text {. }
\end{aligned}
$$

These transformations have been studied geometrically; they have found applications in the theory of congruences of surfaces in $\mathbb{R}^{3}(24)$. The classicists of geometry (Darboux, Tzitzéica, Moutard, see [25], 26]) studied chains of Laplace transformations and carried out a series of useful formal calculations. They observed that the infinite chain of Laplace transformations

$$
\ldots, L_{-1}, L_{0}, L_{1}, L_{2}, \ldots,
$$

where $L_{j+1}=\widetilde{L}_{j}$, is equivalent to a non-linear evolution system, which in the modern literature is called the 'two-dimensionalized Toda chain'. Integrability of it by the methods of soliton theory was discovered in 227, and from the viewpoint of field theory and Lie algebras in [28], [29]. In fact, a series of formal results had already been obtained at the beginning of the 20th century.

We consider the following quantities, that is, the potentials of a chain:

$$
W_{n}=\exp f_{n} .
$$

¿From (74) we obtain

$$
W_{n+1}-W_{n}=e^{f_{n+1}}-e^{f_{n}}=H_{n+1}, \quad H_{n+1}-H_{n}=\frac{1}{2} \partial_{x} \partial_{y}\left(f_{n}\right) .
$$


By means of the substitution $f_{n}=g_{n}-g_{n-1}$ we obtain the 'two-dimensionalized Toda chain'

$$
\frac{1}{2} \square g_{n}=e^{g_{n+1}-g_{n}}-e^{g_{n}-g_{n-1}},
$$

where $\square=\partial_{x} \partial_{y}$.

Darboux posed the problem of classifying the cyclic Laplace chains $L_{N}=L_{0}$ of period $N$. For $N=2$ he deduced, in particular, that solutions of the equation

$$
\square g=\sinh g
$$

define the class of cyclic chains.

His student Tzitzéica (26]) posed the problem: let the operator $L_{0}=L_{N}$ be such that $H_{0}=H_{N}=0$. What kind of non-linear systems can arise in this case? For $N=3$ he showed that such cyclic chains are generated, in particular, by solutions of the equation

$$
\square g=e^{g}-e^{-2 g} .
$$

However, in the hyperbolic case, which was considered by the classicists, there are nothing but formal identities.

Well posed global problems arise only in the elliptic case $\partial_{x} \rightarrow \bar{\partial}, \partial_{y} \rightarrow \partial$, which we also consider in relation to the spectral theory of the Schrödinger operator in electric and magnetic fields for $n=2$, in particular when the fields are smooth non-singular real doubly periodic functions with some lattice of periods $\Gamma$ on the plane $\mathbb{R}^{2}$.

Example 2 Let $N=1$. We have

$$
\begin{array}{r}
L_{1}=L_{0}, \quad H_{1}=H_{0}+\frac{1}{2} \Delta f_{0}, \\
e^{f_{1}}=e^{f_{0}}+H_{1} .
\end{array}
$$

Since $H_{1}=H_{0}$, we obtain $\Delta f_{0}=0$. If $f_{0}$ is a non-singular doubly periodic function (that is, $W_{0} \neq 0$ ), then $f_{0}=$ const.

Since $f_{1}=f_{0}$, we have $H_{1}=0=H_{0}$. Thus, we obtain only a free operator in the zero magnetic field, but this is a consequence of the global hypothesis.

Example 3 Let $N=2$. We have

$$
f_{2}=f_{0}, \quad H_{2}=H_{0} .
$$

Since $H_{1}=H_{0}+\frac{1}{2} \Delta f_{0}, H_{2}=H_{0}=H_{1}+\frac{1}{2} \Delta f_{2}$, we obtain

$$
\frac{1}{2} \Delta\left(f_{0}+f_{1}\right)=0 \text {, }
$$

or

$$
f_{0}=-f_{1}+a
$$


Further, since

$$
\begin{array}{r}
e^{f_{2}}=e^{f_{0}}=e^{f_{1}}+H_{0}, \\
e^{f_{1}}=e^{f_{0}}+H_{1}=e^{f_{0}}+H_{0}+\frac{1}{2} \Delta f_{0},
\end{array}
$$

we obtain

$$
\begin{aligned}
2\left(e^{f_{1}}-e^{f_{0}}\right) & =\frac{1}{2} \Delta f_{0}, \\
2\left(e^{-f_{0}+a}-e^{f_{0}}\right) & =\frac{1}{2} \Delta f_{0} .
\end{aligned}
$$

Let $g=f_{0}-a / 2$. We obtain

$$
\begin{aligned}
2\left(e^{-g+a / 2}+e^{g+a / 2}\right) & =\frac{1}{2} \Delta g, \\
e^{a / 2} \sinh g & =\frac{1}{8} \Delta g .
\end{aligned}
$$

This shows that in the non-singular doubly periodic case all the cyclic Laplace chains of length $N=2$ are described by solutions of the equation

$$
\gamma e^{a / 2} \sinh g=\Delta g .
$$

By stretching the axes $(x, y)$ we reduce it to the sinh-Gordon equation

$$
\Delta g=\sinh g .
$$

Example 4 Let $N=3, H_{0}=0=H_{N}$. Since

$$
H_{0}=H_{3}=H_{2}+\frac{1}{2} \Delta f_{2}=H_{0}+\frac{1}{2} \Delta\left(f_{0}+f_{1}+f_{2}\right),
$$

we obtain for non-singular doubly periodic functions $f_{j}$ :

$$
f_{0}+f_{1}+f_{2}=C .
$$

Since

$$
e^{f_{0}}=e^{f_{3}}=e^{f_{2}}+H_{3}=e^{f_{2}}+H_{0}+\frac{1}{2} \Delta\left(f_{0}+f_{1}+f_{2}\right)=e^{f_{2}}+H_{0},
$$

we come to the conclusion: if $H_{0}=0$, then we have

$$
e^{f_{3}}=e^{f_{2}}=e^{f_{0}} .
$$

Hence it follows that

$$
\begin{aligned}
2 f_{0}+f_{1} & =C, \quad f_{1}=C_{2} f_{0}, \\
e^{f_{2}}=e^{f_{1}}+H_{0}+\frac{1}{2} \Delta\left(f_{0}+f_{1}\right)=e^{f_{0}} & =e^{f_{1}}+\frac{1}{2} \Delta\left(f_{0}+f_{1}\right) .
\end{aligned}
$$


Finally, we have

$$
\begin{array}{r}
e^{f_{0}}-e^{f_{1}}=\frac{1}{2} \Delta\left(f_{0}+f_{1}\right), \\
e^{f_{0}}-e^{C-2 f_{0}}=\frac{1}{2} \Delta\left(f_{0}+C-2 f_{0}\right)=-\frac{1}{2} \Delta f_{0} .
\end{array}
$$

Let $f_{0}=g+a$. We obtain

$$
e^{a} e^{g}-e^{C-2 g-2 a}=-\frac{1}{2} \Delta g .
$$

If $a=C-2 a$, that is, $a=C / 3$, then we finally have

$$
e^{C / 3}\left(e^{g}-e^{-2 g}\right)=-\frac{1}{2} \Delta g,
$$

or, after stretching the scales,

$$
\Delta g=e^{-2 g}-e^{g} .
$$

The following result was proved in [30], [31.

Theorem 2 Suppose we are given a cyclic chain of Laplace transformations

$$
\ldots, L_{0}, L_{1}, L_{2}, \ldots, L_{N}=L_{0}
$$

such that the operators of the chain have smooth doubly periodic real magnetic field and potential. Then all the operators of the chain are topologically trivial and algebro-geometric with respect to the zero level: $L_{j} \psi=0$. The Bloch functions of zero level with all complex quasimomenta are defined and sweep out a Riemann surface of finite genus; the coefficients of the operators $L_{j}$ are expressed in terms of theta-functions of this surface (the 'Fermi-curve'), which is common for all operators of the chain.

We recall that two-dimensional algebro-geometric operators were introduced and studied in [32, [33]; for a review of their theory see [34].

The idea of the proof of this theorem can be seen for $N=2$, where the requirement of cyclicity of the chain under the conditions of the theorem reduces to the equation $\Delta g=\sinh g$.

In studying surfaces with the topology of a torus in $\mathbb{R}^{3}$ with constant mean curvature it was established that all doubly periodic non-singular solutions of this equation are finite-zone (algebro-geometric); see the survey [35]. Finally, this follows from the fact that any manifold of solutions of a non-linear elliptic equation on a torus (compact manifold) has finite dimension at a non-singular point. Further arguments, which lead to the finite-zone property of solutions of the equation $\Delta g=\sinh g$, follow the standard scheme that was already developed in 1974 for the KdV equation (see the survey [2]); commutative fluxes are linearly dependent on a finite-dimensional invariant submanifold. 
A natural generalization of this idea for all $N \geq 2$ also leads to the proof of the theorem, as was noted by the authors of [30], [31].

Definition 7 A semicyclic Laplace chain is a set of Schrödinger operators

$$
L_{0}, \ldots, L_{N}, \quad L_{j+1}=\widetilde{L}_{j}
$$

such that $L_{N}=L_{0}+C$.

Definition 8 A quasicyclic Laplace chain is a set of Schrödinger operators

$$
L_{0}, \ldots, L_{N}, \quad L_{j+1}=\widetilde{L}_{j}
$$

such that the extreme operators are completely factorized with a constant potential:

$$
\begin{array}{r}
2 L_{0}=Q_{01} Q_{02}+2 V_{0}, \quad 2 L_{N}=Q_{N 1} Q_{N 2}+2 V_{N}, \\
V_{0}=\mathrm{const}, \quad V_{N}=\mathrm{const}, \\
Q_{j 2}=\bar{\partial}+B_{j}(z, \bar{z}), \quad Q_{j 1}=\partial+A_{j}(z, \bar{z}) .
\end{array}
$$

¿From the definition we have

$$
\begin{array}{ll}
2 L_{j}=Q_{j 2} Q_{j 1}+2 W_{j}, \quad & W_{0}=-H_{0}, \quad W_{N}=-H_{N}, \\
& 2 \widetilde{L}_{j}=W_{j} Q_{j 1}^{+} W_{j}^{-1} Q_{j 2}+2 W_{j} .
\end{array}
$$

Example $5 N=1$. A semicyclic chain of length 1. We have

$$
\begin{array}{r}
2 L_{1}=2 L_{0}-2 C, \quad H_{1}=H_{0}, \\
e^{f_{1}}=e^{f_{0}}+C=e^{f_{0}}+H_{1}, \quad H_{1}=C .
\end{array}
$$

Hence we obtain

$$
\Delta f_{0}=0 .
$$

In the non-singular doubly periodic case it follows that

$$
f_{0}=\text { const }, \quad H=C .
$$

Hence we arrive at a Landau operator in a homogeneous constant magnetic field $H=C$ and zero potential. Starting from the solutions (let $C>0$ )

$$
Q_{02} \psi_{0}=0, \quad L_{0} \psi_{0}=0, \quad V_{0}=0,
$$

we obtain all the Landau levels

$$
\begin{array}{r}
\left(Q_{01}\right) \psi_{0}=\psi_{1} \\
2 L_{1} \psi_{1}=0 \Longrightarrow 2 L_{0} \psi_{1}=2 C \psi_{1}, C=H
\end{array}
$$

Hence, from the lowest level $Q_{0} \psi_{0}=0$ we obtain the first level $\psi_{1}=Q_{0}^{+} \psi_{0}$. These spaces of solutions are infinite-dimensional on every level, called 'Landau levels' for the Landau operator

$$
H+2 L_{0}=Q_{02} Q_{01}+H, \quad Q_{02} \psi_{0}=0 .
$$


Its levels are

$$
\lambda_{n}=H+2 n H, \quad \psi_{n}=\left(Q_{01}\right)^{n} \psi_{0} .
$$

Example $6 N=2$. A semicyclic chain of length 2. Here we have $H_{2}=H_{0}$. In the non-singular doubly periodic case, from the equality

$$
H_{2}=H_{0}+\frac{1}{2} \Delta\left(f_{0}+f_{1}\right)
$$

it follows that

$$
\Delta\left(f_{0}+f_{1}\right)=0 \Longrightarrow f_{0}+f_{1}=a .
$$

Since

we obtain

$$
\begin{array}{r}
e^{f_{2}}=e^{f_{0}}+C=e^{f_{1}}+H_{2}=e^{f_{1}}+H_{0}, \\
e^{f_{1}}=e^{f_{0}}+H_{0}+\frac{1}{2} \Delta f_{0},
\end{array}
$$

$$
\begin{array}{r}
e^{f_{0}}-e^{f_{1}}=H_{0}-C, \\
-e^{f_{1}}+e^{f_{0}}=-H_{0}-\frac{1}{2} \Delta f_{0} .
\end{array}
$$

Finally, we have

$$
\begin{array}{r}
2\left(e^{f_{0}}-e^{f_{1}}\right)=-C-\frac{1}{2} \Delta f_{0}, \\
f_{1}=a-f_{0} .
\end{array}
$$

Thus we arrive at the equation

$$
e^{f_{0}}-e^{a-f_{0}}+\frac{C}{2}=-\frac{1}{4} \Delta f_{0} .
$$

Let $f_{0}=g+a / 2$. We have the equality

$$
\begin{array}{r}
e^{g+a / 2}-e^{a / 2-g}+\frac{C}{2}=-\frac{1}{4} \Delta g, \\
-2 C-2 e^{a / 2} \sinh g=\Delta g .
\end{array}
$$

This equation has an extensive family of doubly periodic non-singular real solutions.

Solutions of the equation $L_{N} \psi_{N}=\left(L_{0}+C\right) \psi_{N}=0$ are obtained from solutions of the equation $L_{0} \psi_{0}=0$ by the chain of algebraic 'creation operators'

$$
\psi_{N}=Q_{N-1,2} \cdots Q_{0,2}\left(\psi_{0}\right), \quad L_{0} \psi_{N}=(-C) \psi_{N} .
$$

Hence, the levels of spectrum $\varepsilon=0$ and $\varepsilon=-C$ for the operator $L_{0}$ are connected by these operators and are obtained from each other. It is easy to see that magnetic Bloch solutions are transformed into magnetic Bloch ones under the action of these operators if the magnetic flux is quantized (that is, the Bloch solutions are defined). 
Lemma 9 If $C \neq 0$, then the magnetic flux of a non-singular semicyclic chain with potentials that do not vanish is different from zero.

Remark 6 Since $H_{j+1}=H_{j}+\frac{1}{2} \Delta\left(f_{j}\right)$ and $f_{j}$ is non-singular, we see that the fluxes of the operators $L_{j}$ coincide: $\left[H_{j+1}\right]=\left[H_{j}\right]$.

Proof of the Lemma Since

$$
e^{f_{N}}=e^{f_{N-1}}+H_{0}+\frac{1}{2} \Delta\left(\sum_{j=0}^{N-1} f_{j}\right)=e^{f_{0}}+C,
$$

and $\left[e^{f_{N}}\right]=\left[e^{f_{N-1}}\right]+[H]$, we obtain

$$
\left[e^{f_{N}}\right]=\left[e^{f_{0}}\right]+[C]=\left[e^{f_{0}}\right]+K C=\left[e^{f_{0}}\right]+N[H] .
$$

Hence, for the flux $N[H]=N\left[H_{0}\right]=K C$ it follows that

$$
C=\frac{N[H]}{K},
$$

where $K$ is the area of an elementary cell. We have proved Lemma 9.

(For purely cyclic non-singular chains we have $C=0$, that is, the flux is always equal to zero.)

The greatest interest is in quasicyclic chains, with which the main results of [30], 31] are connected.

Theorem 3 Let the quasicyclic Laplace chain of the Schrödinger operators

$$
\begin{array}{r}
L_{0}, \ldots, L_{N}, \quad L_{j+1}=\widetilde{L}_{j}, \\
2 L_{0}=Q_{0,1}^{+} Q_{0,2}, \quad 2 L_{N}=Q_{N, 1}^{+} Q_{N, 2}+2 C_{N}
\end{array}
$$

be given with doubly periodic real quantities $\left(f_{j}, H_{j}\right)$, and suppose that the magnetic flux $\left[H_{j}\right]$ is positive, $[H]>0$. Then the operator $L_{N}$ has two strongly degenerate discrete levels, which are isomorphic to the Landau levels and one of them is the lowest:

a) $L_{N} \psi=C_{N} \psi, Q_{N} \psi=0, \lambda=C_{N}$,

b) $L_{N} \psi=0, \lambda=0$.

In addition, $C_{N}=N[H] / K$, where $K$ is the area of an elementary cell.

Here $Q_{j 2}=\bar{\partial}+B_{j}(z, \bar{z}), Q_{j 1}=\partial+A_{j}(z, \bar{z})$.

Proof First, solving the equation

$$
Q_{N, 2} \psi=0
$$


according to the scheme of [31], we obtain the eigenvalue $L_{N} \psi=C_{N} \psi$. If $[H]>0$, such a solution exists and gives a huge space isomorphic to the Landau level according to [7], [8], 36. A basis in this space, mentioned in [7], 8], [36], is the magnetic Bloch basis, which is suitable only in the case of the integer-valued flux $[H]=2 \pi m>0$; however, the result itself is true for all positive values of the flux.

We obtain the second level as follows. We solve the equation $Q_{02} \psi=0$ according to [7], [8] and obtain also for $[H]>0$ a huge space of functions $\psi$ isomorphic to the Landau level, which can be defined by using elliptic formulae for the integer-valued flux $[H]$, common for $H_{0}$ and $H_{N}$,

$$
[H]=\left[H_{0}\right]=\left[H_{N}\right]
$$

Next we factorize the operator $L_{0}$ in the form

$$
2 L_{0}=Q_{0,2} Q_{0,1}+2 H_{0}=Q_{0,1}^{+} Q_{0,2} \text {. }
$$

Then we apply the creation operator $N$ times to a solution of $Q_{0} \psi=0$ :

$$
\widehat{\psi}=Q_{N-1,1} \circ \cdots \circ Q_{0,1} \psi \text {. }
$$

The new function $\widehat{\psi}$ satisfies the equation

$$
L_{N} \widehat{\psi}=0=\left(Q_{N, 1} Q_{N, 2}+C_{N}\right) \widehat{\psi} .
$$

The function $\widehat{\psi}$ belongs to $\mathcal{L}_{2}(\mathbb{R})$ if $\psi$ belongs to $\mathcal{L}_{2}(\mathbb{R})$.

Now we find the number $C_{N}$. We have for the Laplace chain the following boundary conditions:

$$
-e^{f_{0}}=H_{0}, \quad-e^{f_{N}}=H_{N}+C_{N} .
$$

For the mean values we obtain

$$
\left[e^{f_{j+1}}\right]=\left[e^{f_{j}}\right]+\left[H_{j+1}\right]=\left[e^{f_{j}}\right]+[H]=\left[e^{f_{0}}\right]+(j+1)[H]=(j+2)[H] .
$$

In particular, for $j+1=N$ we obtain

$$
e^{f_{N}}=[H]+\left[C_{N}\right]=(N+1)[H]
$$

where $\left[C_{N}\right]=K C_{N}$ or $C_{N}=N[H] / K$.

Remark 7 Let the operator $L$ be given in the self-adjoint form,

$$
\begin{array}{r}
Q=Q_{1}=\partial+A, \quad Q_{2}=-Q^{+}=\bar{\partial}+B, \\
\bar{B}=-A .
\end{array}
$$

It is also convenient to choose the 'Lorentz gauge', for which all the formulae are written in [7], [8]:

$$
\operatorname{Im}\left(A_{\bar{z}}\right)=0 .
$$


Then we have

$$
2 L=-Q^{+} Q+2 W=-Q Q^{+}+2 V .
$$

Further, if $L \psi=0$, we carry out the Laplace transformation together with the gauge transformation

$$
\begin{array}{r}
L \longmapsto e^{g} \widetilde{L} e^{-g}, \quad W=e^{f}, \\
\widetilde{L}=-W Q W^{-1} Q^{+}+2 W, \\
\widetilde{\psi} \longmapsto e^{g} Q \psi=e^{g} \widetilde{\psi} .
\end{array}
$$

Choosing $g=-f / 2$, we obtain the operator $\widetilde{\widetilde{L}}=e^{-f / 2} \widetilde{L} e^{f / 2}$ in the form

$$
\widetilde{\widetilde{L}}=e^{-f / 2} \widetilde{L} e^{f / 2}=-\widetilde{Q} \widetilde{Q}^{+}+2 W,
$$

where $\widetilde{Q}=e^{-f / 2} Q e^{f / 2}, \widetilde{Q}^{+}=e^{f / 2} Q^{+} e^{-f / 2}$. We obtain explicitly the selfadjoint operator in the Lorentz gauge transformation.

Example 7 Let $N=1$. From the definition for $L_{0}$ we have

$$
2 L_{0}=-Q_{0}^{+} Q_{0}=-Q_{0} Q_{0}^{+}+2 H_{0}
$$

where

$$
Q_{0}=\partial+A, \quad-Q_{0}^{+}-\bar{\partial}+B, \quad H_{0}=W_{0}=e^{f_{0}} .
$$

Therefore

$$
2 L_{1}=-\left(e^{f_{0} / 2} Q_{0}^{+} e^{f_{0} / 2}\right)\left(e^{f_{0} / 2} Q_{0} e^{-f_{0} / 2}\right)+2 H_{0}={\widetilde{L_{0}}}_{0}=e^{-f_{0} / 2} \widetilde{L}_{0} e^{f_{0} / 2} .
$$

¿From the quasicyclicity condition for $N=1$ we have

$$
H_{0}=\text { const }, \quad L_{1}=L_{0}+2 H_{0} .
$$

We arrive at the Landau operator.

Example 8 Let $N=2$.

$$
\begin{array}{r}
2 L_{0}=-Q_{0}^{+} Q_{0}=-Q_{0} Q_{0}^{+}+2 H_{0}, \quad H_{0}=e^{f_{0}}, \\
2 L_{2}=-Q_{2}^{+} Q_{2}+2 C_{2}=-Q_{2} Q_{2}^{+}+2 C_{2}+2 H_{2}, \\
e^{f_{2}}=C_{2}+H_{2}=e^{f_{1}}+H_{2} .
\end{array}
$$

We see that $e^{f_{1}}=$ const. Further,

$$
C_{2}=e^{f_{1}}=e^{f_{0}}+H_{1}=e^{f_{0}}+H_{0}+\frac{1}{2} \Delta f_{0},
$$


where $H_{0}=e^{f_{0}}$. Hence we have

$$
\begin{gathered}
C_{2}-2 e^{f_{0}}=\frac{1}{2} \Delta f_{0}, \\
2 C_{2}=-4 e^{f_{0}}=\Delta f_{0} .
\end{gathered}
$$

Putting $f_{0}=g+a$, where $e^{a}=C_{2} / 2$, we obtain $\Delta g=e^{a}\left(1-e^{g}\right)$. By stretching the axes we bring this equation to the form

$$
\Delta g=1-e^{g} .
$$

The last equation has an extensive family of smooth real doubly periodic solutions. Even its solutions, dependent on one variable (for example, on $x$ ), which can be found by quadrature, give us a continuous family belonging to this class, with an arbitrary period in $y$ :

$$
\begin{array}{r}
f_{0}^{\prime \prime}=2 C_{2}-4 e^{f_{0}}, \quad C_{2}>0, \\
\tau^{\prime \prime}=-\frac{\partial}{\partial \tau}(U(\tau)), \\
U(\tau)=-A \tau+4 e^{\tau}, \quad \tau=f_{0}, \quad A=2 C_{2} .
\end{array}
$$

The dependence of $f_{0}(x)$ is determined by quadrature:

$$
\begin{aligned}
x-x_{0} & =\int \frac{d \tau}{\sqrt{-4 e^{\tau}+A \tau+E}}, \\
E & \geq E_{\min }=A\left(1-\log \frac{A}{4}\right) .
\end{aligned}
$$

Equation (82) has occurred in various physical problems - in plasma theory, superconductivity theory, and so on. Hector de Vega informed one of the authors (S. P. Novikov) that equation (82) arose as the Bogomol'nyi reduction for the Ginzburg-Landau equation for the critical value of the parameter which separates superconductors of types I and II (see [37]). However, the application of this form of the Ginzburg-Landau equation at a critical point is not clear. In addition, the magnetic field in this model differs from ours by a non-zero constant, and in the theory of superconductors solutions with singularities are necessary.

Hypothesis For periods $N \geq 5$ the quasiperiodicity condition does not give smooth real doubly periodic solutions, apart from a constant. In any case, the constant (the Landau operator) does not allow any variation - it is an isolated solution.

According to the hypothesis of Novikov, the resulting class of operators is 'maximally exactly soluble' among two-dimensional Schrödinger operators with smooth real doubly periodic electric potential and magnetic field with a non-zero (let it be integer-valued) flux $[H]=2 \pi m$. 
These operators have two discrete levels with infinite degeneration that are isomorphic to the Landau levels. One of those levels is basic (that is, the lowest one) and the second one is certainly not the nearest to the first one (at least one 'magnetic zone' lies between them). If there are three strongly degenerate discrete levels, then, as we suppose, the operator will coincide with the Landau operator.

The general arrangement of the spectrum of Schrödinger operators in a magnetic field with integer-valued flux, interesting aspects of the inverse spectral problem, and geometric and topological properties of the spectrum of these operators were discussed in [15], 38], [39].

If the magnetic flux is quantized, then we have the 'magnetic Bloch function' of the dispersion law with number $m \geq 0, m \in \mathbb{Z}$

$$
\widehat{T}_{1} \psi=e^{i p_{1} T_{1}} \psi, \quad \widehat{T}_{2} \psi=e^{i p_{2} T_{2}} \psi, \quad L \psi_{m}=\varepsilon_{m}\left(p_{1}, p_{2}\right) \psi_{m},
$$

$\psi=\psi_{m}\left(x, y, p_{1}, p_{2}\right),[H]=\Phi \in 2 \pi \mathbb{Z}, \Phi \neq 0$.

For definiteness, let the function $\psi$ be normalized by analogy with finite-zone theory [2]:

$$
\left.\psi_{m}\left(x, y, p_{1}, p_{2}\right)\right|_{x=x_{0}, y=y_{0}}=1 .
$$

The function $\psi_{m}\left(x, y, x_{0}, y_{0}, p_{1}, p_{2}\right)$ under the fixed normalization $\left(x_{0}, y_{0}\right)$ is defined on the 'phase space'

$$
\left(x, y, p_{1}, p_{2}\right) \in \mathbb{R}^{2} \times \mathbb{T}^{2} .
$$

Moreover, the function $\psi$ is defined up to normalization

$$
\psi_{n} \mapsto f\left(p_{1}, p_{2}\right) \psi_{n} .
$$

The manifold of zeros of the function $\psi_{m}, \psi_{m}=0$, splits into two parts:

1) 'topological zeros', where $\psi_{m}(x, y) \equiv 0$ for fixed $p_{1}, p_{2}$; this part of the zeros changes under transformations (85), but the 'algebraic number of zeros' is the 'Chern class' $c_{1}^{(m)}$ of the dispersion law with number $m$ for the operator $L$ of general position, where the dispersion laws do not intersect,

$$
\varepsilon_{m_{1}}\left(p_{1}, p_{2}\right) \neq \varepsilon_{m_{2}}\left(p_{1}, p_{2}\right), \quad m_{1} \neq m_{2} ;
$$

2) 'geometric zeros', where locally at a general point we can assume that

$$
\left(p_{1}, p_{2}\right)=\gamma_{m}(x, y), \quad m \geq 0 .
$$

As $m \rightarrow \infty$ the dispersion laws converge to constants:

$$
\varepsilon_{m}\left(p_{1}, p_{2}\right) \rightarrow \text { const }=(2 m+1)[H],
$$

analogous to the forbidden zones of the one-dimensional periodic Schrödinger operator.

Problem Find the analogue of the 'Dubrovin equations' for the set $\left\{\gamma_{m}(x, y)\right\}$ for all $m$; solve the inverse problem of reconstructing the operator $L$, starting from the set of geometric data, in the phase space (84), analogous to the onedimensional data, without entering into the complex domain of quasimomenta. 


\section{$\S 5$. Difference equations and Laplace transforma- tions. The hyperbolic case}

The simplest hyperbolic difference equation on the standard quadratic lattice $\Gamma$ in $\mathbb{R}^{2}$ is

$$
a_{n} \psi_{n}+b_{n} \psi_{n+T_{1}}+c_{n} \psi_{n+T_{2}}+d_{n} \psi_{n+T_{1}+T_{2}}=0,
$$

where $n=\left(n_{1}, n_{2}\right)$ is the number of a lattice point, $n_{j} \in \mathbb{Z}, T_{1}=(1,0)$, $T_{2}=(0,1)$ are the basis vectors of the lattice. We also denote by $T_{1}, T_{2}$ the basis operators of translations which act on functions defined on $\mathbb{Z}^{2}$ :

$$
\begin{gathered}
T_{1} \psi\left(n_{1}, n_{2}\right)=\psi\left(n_{1}+1, n_{2}\right), \\
T_{2} \psi\left(n_{1}, n_{2}\right)=\psi\left(n_{1}, n_{2}+1\right) .
\end{gathered}
$$

We can write (86) in the following way:

$$
L \psi=\left(a_{n}+b_{n} T_{1}+c_{n} T_{2}+d_{n} T_{1} T_{2}\right) \psi=0 .
$$

It was already observed in [40] that for an equation of this form there is a class of algebro-geometric exactly soluble cases with effective theta-function formulae.

The operator $L$ is defined up to multiplication by a function: $L \mapsto f_{n} L$. We also have gauge transformations, and so together we have a system of the following equivalences of (87):

$$
L \mapsto f_{n} L g_{n}, \quad \psi_{n} \mapsto g_{n}^{-1} \psi_{n},
$$

where $f_{n}, g_{n}$ are non-vanishing functions on the lattice.

Lemma 100 The operator $L$ admits a unique representation in the form

$$
\text { a) } L=f_{n}\left(\left(1+u_{n} T_{1}\right)\left(1+v_{n} T_{2}\right)+w_{n}\right)
$$

or in the form

$$
\text { b) } L=f_{n}^{\prime}\left(\left(1+v_{n}^{\prime} T_{2}\right)\left(1+u_{n}^{\prime} T_{1}\right)+w_{n}^{\prime}\right) \text {. }
$$

The coefficients $u_{n}, v_{n}, w_{n}, f_{n}$ for the factorization a) are determined from the formulae

$$
a_{n}=f_{n}\left(1+w_{n}\right), \quad f_{n} u_{n}=b_{n}, \quad f_{n} v_{n}=c_{n}, \quad f_{n} u_{n} v_{n+T_{1}}=d_{n} .
$$

The proof is based on direct verification.

Using both factorizations a) and b), we define two Laplace transformations of (87) $L \psi=0$.

Definition 9 A Laplace transformation of the first type is a transformation

$$
\begin{aligned}
L \longmapsto \widetilde{L} & =\widetilde{f}_{n}\left(w_{n}\left(1+v_{n} T_{2} w_{n}^{-1}\right)\left(1+u_{n} T_{1}\right)+w_{n}\right), \\
\psi \longmapsto \widetilde{\psi} & =\left(1+v_{n} T_{2}\right) \psi .
\end{aligned}
$$


A Laplace transformation of the second type is a transformation

$$
\begin{aligned}
& L \longmapsto \widetilde{L}^{\prime}=\widetilde{f}^{\prime}\left(w_{n}^{\prime}\left(1+u_{n}^{\prime} T_{1}\right)\left(w_{n}^{\prime}\right)^{-1}\left(1+v_{n}^{\prime} T_{2}\right)+w_{n}^{\prime}\right), \\
& \psi \longmapsto \widetilde{\psi}^{\prime}=\left(1+u_{n}^{\prime} T_{1}\right) \psi .
\end{aligned}
$$

Lemma 111 The Laplace transformations of the first and second types are mutually inverse.

The proof is obtained by simple substitution, as in the continuous case.

We denote a Laplace transformation of the first type by $\Lambda_{12}^{++}$and of the second type by $\Lambda_{21}^{++}$. Lemma 11 claims that $\Lambda_{12}^{++} \Lambda_{21}^{++}=1$.

It is appropriate to note that in a completely analogous way we can define the Laplace transformation of (86), which corresponds to an arbitrary pair of orthonormal basis translations

$$
\begin{aligned}
\left(T_{1}, T_{2}\right),\left(T_{2}, T_{1}\right) & \Lambda_{12}^{++}, \Lambda_{21}^{++}, \\
\left(T_{1}^{-1}, T_{2}\right),\left(T_{2}, T_{1}^{-1}\right) & \Lambda_{12}^{-+}, \Lambda_{21}^{+-}, \\
\left(T_{1}, T_{2}^{-1}\right),\left(T_{2}^{-1}, T_{1}\right) & \Lambda_{12}^{+-}, \Lambda_{21}^{-+}, \\
\left(T_{1}^{-1}, T_{2}^{-1}\right),\left(T_{2}^{-1}, T_{1}^{-1}\right) & \Lambda_{12}^{--}, \Lambda_{21}^{--} .
\end{aligned}
$$

Thus, we have eight Laplace transformations $\Lambda_{12}^{\varepsilon \sigma}, \Lambda_{21}^{\varepsilon \sigma}, \varepsilon, \sigma= \pm$, and

$$
\Lambda_{12}^{\varepsilon \sigma} \Lambda_{21}^{\sigma \varepsilon}=1 .
$$

Hence, the Laplace transformations here form a group with four generating elements $\Lambda_{12}^{\varepsilon \sigma}$, the structure of which we do not know so far.

Now we return to the transformation $\Lambda_{12}^{++}$, described above. The Laplace transformations are well defined on the equivalence classes (88). Therefore, as in the continuous case, they should be registered on the invariants of the equivalence classes, which are analogous to the magnetic field and electric potential.

Lemma 122 The complete set of invariants with respect to the equivalence (88) is given by the following basis functions:

$$
K_{1 n}=\frac{b_{n} c_{n+T_{1}}}{d_{n} a_{n+T_{1}}}, \quad K_{2 n}=\frac{c_{n} b_{n+T_{2}}}{d_{n} a_{n+T_{2}}} .
$$

All other invariants are expressed in terms of $K_{1 n}, K_{2 n}$ and their translations on the lattice.

Proof The proof of the invariance of these quantities is straightforward: it is a direct elementary verification. It is convenient to carry out the proof of their completeness for the factorized form (89). Expressing $a_{n}, b_{n}, c_{n}, d_{n}$ in terms of $f_{n}, u_{n}, v_{n}, w_{n}$, we obtain

$$
K_{1 n}=\frac{1}{1+w_{n}}, \quad K_{2 n}=\frac{1}{1+w_{n+T_{2}}}\left(v_{n} u_{n+T_{2}} v_{n+T_{1}}^{-1} u_{n}^{-1}\right) .
$$


It is clear that the potential $w_{n}$ is gauge invariant, and the function $f_{n}$ can be shortened in equivalence classes. Hence, everything reduces to gauge equivalence for $f_{n}=1$. Here the 'potential' $w_{n}$ and the 'curvature'

$$
H=v_{n} u_{n}^{-1} u_{n+T_{2}} v_{n+T_{1}}^{-1}=\frac{v_{n} u_{n+T_{2}}}{u_{n} v_{n+T_{1}}}
$$

are gauge invariant. We need to find all gauge invariants of the operator

$$
L_{0}=\left(1+u_{n} T_{1}\right)\left(1+v_{n} T_{2}\right)
$$

with respect to the transformations

$$
L_{0} \longmapsto \tau_{n}\left(1+u_{n} T_{1}\right)\left(1+v_{n} T_{2}\right) \tau_{n}^{-1}=\left(1+u_{n} \frac{\tau_{n}}{\tau_{n+T_{1}}} T_{1}\right)\left(1+v_{n} \frac{\tau_{n}}{\tau_{n+T_{2}}} T_{2}\right) .
$$

This problem is easily reduced to the classification of gauge invariant monomials, that is, products of the quantities $u_{n+k T_{1}+l T_{2}}, v_{n+k T_{1}+l T_{2}}$ for different (finitely many) $k, l$. Elementary combinatorics leads to the conclusion that all these monomials are products of monomials (94) and their translations in some powers. We omit these details.

We use the basis of invariants $w_{n}, H_{n}$, by analogy with the continuous case.

Lemma 133 The Laplace transformation for invariants has the form

$$
\begin{gathered}
1+\widetilde{w}_{n+T_{1}}=\left(1+w_{n+T_{2}}\right) \frac{w_{n} w_{n+T_{1}+T_{2}}}{w_{n+T_{1}} w_{n+T_{2}}} H_{n}^{-1}, \\
\widetilde{H}_{n}=\frac{1+w_{n+T_{2}}}{1+\widetilde{w}_{n+T_{2}}} .
\end{gathered}
$$

The proof is obtained by substituting the expressions for $\widetilde{K}_{1}, \widetilde{K}_{2}$ in terms of $\widetilde{a}, \widetilde{b}, \widetilde{c}, d$.

We now consider an infinite chain of Laplace transformations for the quantities

$$
\widetilde{H}_{n}^{(k)}=H_{n}^{(k+1)}, \quad \widetilde{w}_{n}^{(k)}=w_{n}^{(k+1)},
$$

where $n=\left(n_{1}, n_{2}\right), k \in \mathbb{Z}$, and $H_{n}^{(k+1)}, w_{n}^{(k+1)}$ are obtained from the previous ones by a Laplace transformation. Eliminating $H_{n}^{(k)}$ from the equations, we obtain a 'completely discrete analogue of the two-dimensionalized Toda chain':

$$
\frac{1+w_{n+T_{1}}^{(k+2)}}{1+w_{n+T_{1}}^{(k+1)}} \frac{1+w_{n+T_{2}}^{(k+1)}}{1+w_{n+T_{2}}^{(k)}}=\frac{w_{n+T_{1}}^{(k)} w_{n+T_{2}}^{(k)}}{w_{n}^{(k)} w_{n+T_{1}+T_{2}}^{(k)}} .
$$

Problem Find a comparison between this completely discrete analogue of the two-dimensionalized Toda chain and the systems studied in [41], starting from the theory of the 'Yang-Baxter' equation. 
Example 9 We consider a cyclic chain of length $N=2$. After simple calculations we obtain

$$
\begin{array}{r}
w_{n}^{(1)}=C\left(w_{n}^{(0)}\right)^{-1}, \\
w_{n+T_{1}+T_{2}}=w_{n}^{-1}\left(C+w_{n+T_{1}}\right)\left(C+w_{n+T_{2}}\right)\left(1+w_{n+T_{1}}\right)^{-1}\left(1+w_{n+T_{2}}\right)^{-1} .
\end{array}
$$

Finally, we note that there is, as we showed above, the group of all hyperbolic Laplace transformations, generated by four basis transformations $\Lambda_{12}^{\varepsilon \sigma}, \varepsilon, \sigma= \pm$, which corresponds to the choice of the bases $\left(T_{1}^{\varepsilon}, T_{2}^{\sigma}\right)$. In principle, to each word in this group corresponds the condition for cyclicity of a chain. However, the structure of this group is not known so far.

\section{§6. The Laplace transformation for elliptic two- dimensional difference operators on a regular lat- tice. Equations of a triangle, curvature}

It appears that in the elliptic case Laplace transformations cannot be defined for difference operators of the second order on a square lattice. To give their correct definition we should follow two principles.

I. In order that continuous and discrete 'spectral symmetries' of continuous Schrödinger operators be conserved in the difference case, all translations should be considered as covariant, as in the one-dimensional case (see §3).

II. In the two-dimensional case for elliptic operators we should replace the square lattice by an equilateral triangular lattice, where the following translations have the same length:

$$
T_{1}^{ \pm 1}, T_{2}^{ \pm 1},\left(T_{1} T_{2}\right)^{ \pm 1}
$$

We denote an arbitrary pair of neighbouring translations (97) (which go anticlockwise) by $T_{1}^{*}, T_{2}^{*}$ and ascribe to this pair a Laplace transformation generated by factorization

$$
L=\left(x_{n}+y_{n} T_{1}^{*}+z_{n} T_{2}^{*}\right)\left(x_{n}+y_{n-T_{1}^{*}} T_{1}^{*-1}+z_{n-T_{2}^{*}} T_{2}^{*-1}\right)+w_{n},
$$

where the self-adjoint operator $L$ has the form

$$
L=a_{n}+b_{n} T_{1}+c_{n} T_{2}+d_{n-T_{2}} T_{1} T_{2}^{-1}+b_{n-T_{1}} T_{1}^{-1}+c_{n-T_{2}} T_{2}^{-1}+d_{n-T_{1}} T_{2} T_{1}^{-1},
$$

all the coefficients are real, and $b_{n}, c_{n}, d_{n} \in \mathbb{R}_{+}$. It is easy to see that the coefficient $a_{n}$ is a numerical function on $\mathbb{Z}^{2}$, while the coefficients $b_{n}, c_{n}, d_{n}$ are a connection sitting on the corresponding edges of the lattice. As a result the operator contains an interaction of each vertex with all six nearest neighbours, and all edges have the same length. We start from the following observation (42], 43]). 
Lemma 14 4 A real self-adjoint operator of the form (99) with non-zero coefficients $b_{n}, c_{n}, d_{n}$ always admits a unique representation in the form

$$
L=Q Q^{+}+w_{n}, \quad Q=x_{n}+y_{n} T_{1}+z_{n} T_{2},
$$

where $T_{1}^{+}=T_{1}^{-1}, T_{2}^{+}=T_{2}^{-1},(A B)^{+}=B^{+} A^{+}$. Moreover we have the equalities

$$
\begin{aligned}
x_{n} y_{n-T_{1}} & =b_{n-T_{1}}, \\
x_{n} z_{n-T_{2}} & =c_{n-T_{2}}, \\
y_{n-T_{1}} z_{n-T_{2}} & =d_{n-T_{1}-T_{2} .}
\end{aligned}
$$

In all, following [42, 443, we obtain six different factorizations, which correspond to the following six pairs of periods $\left.\left.\left(T_{1}^{*}, T_{2}^{*}\right): 1\right)\left(T_{1}, T_{2}\right), 2\right)\left(T_{2}, T_{1}^{-1} T_{2}\right)$, 3) $\left.\left.\left.\left(T_{1}^{-1} T_{2}, T_{1}^{-1}\right), 4\right)\left(T_{1}^{-1}, T_{2}^{-1}\right), 5\right)\left(T_{2}^{-1}, T_{2}^{-1} T_{1}\right), 6\right)\left(T_{2}^{-1} T_{1}, T_{1}\right)$,

$$
L=Q_{j} Q_{j}^{+}+w_{j n},
$$

$j=1,2,3,4,5,6 . j$ is determined modulo 6 .

Definition 10 A Laplace transformation $P_{j}$ of type $j$ is as follows:

$$
\begin{aligned}
& P_{j} L \longmapsto \widetilde{L}^{(j)}=w_{j n}^{1 / 2} Q_{j}^{+} w_{j n}^{-1} Q_{j} w_{j n}^{1 / 2}+w_{j n}, \\
& P_{j} \psi \longmapsto \widetilde{\psi}=w_{j n}^{-1 / 2} Q_{j}^{+} \psi .
\end{aligned}
$$

It is defined if $w_{j n}>0$.

Lemma 155 The Laplace transformations $P_{j}$ and $P_{j+3}$ are mutually inverse. The others are connected by the following relations:

$$
\begin{aligned}
& w_{1 n}=w_{3 n}=w_{5 n} \text {, } \\
& Q_{1}=Q_{3} T_{1}=Q_{5} T_{2}, \\
& \widetilde{L}^{(1)}=\sqrt{\frac{w_{n}}{w_{n-T_{1}}}} T_{1}^{-1} \widetilde{L}^{(3)} T_{1} \sqrt{\frac{w_{n}}{w_{n-T_{1}}}}=\sqrt{\frac{w_{n} w_{n-T_{2}}}{T_{2}}} T_{2}^{-1} \widetilde{L}^{(5)} T_{2} \sqrt{\frac{w_{n}}{w_{n-T_{2}}}} .
\end{aligned}
$$

In the equivalence class

$$
L \mapsto f_{n} L f_{n}
$$

the operators $\widetilde{L}^{(1)}, \widetilde{L}^{(3)}, \widetilde{L}^{(5)}$ are unitarily adjoint.

For $j=2,4,6$ analogous formulae hold:

$$
\widetilde{L}^{(4)}=\sqrt{\frac{w_{n}}{w_{n+T_{1}}}} T_{1} \widetilde{L}^{(6)} T_{1}^{-1} \sqrt{\frac{w_{n}}{w_{n+T_{1}}}}=\sqrt{\frac{w_{n}}{w_{n+T_{2}}}} T_{2} \widetilde{L}^{(2)} T_{2}^{-1} \sqrt{\frac{w_{n}}{w_{n+T_{2}}}} .
$$

Remark 8 In the definition of Laplace transformations we can discard the requirement $w_{n}>0$, replacing it by the condition $w_{n} \neq 0$, and set

$$
\widetilde{L}^{(j)}=Q_{j}^{+} w_{j n}^{-1} Q_{j}+1, \quad \widetilde{\psi}=Q_{j}^{+} \psi .
$$


We obtain the same transformations up to equivalence (104), but $P_{j}$ and $P_{j+3}$ are not exactly mutually inverse. We note that in the discrete case, as opposed to the continuous one, the condition $w \neq 0$ does not mean that $w$ has constant sign.

Remark 9 The relations (103) were not noted in 442-444.

Remark 10 If the potential $w_{n} \equiv w_{0}$ is constant (for example, for the first factorization), then the Laplace transformation

$$
P_{1} L \mapsto \widetilde{L}^{(1)}
$$

acts on the eigenfunctions of all levels $L \psi=\lambda \psi$ :

$$
P_{1} \psi=Q_{1}^{+} \psi, \quad P_{1} L=Q_{1}^{+} Q_{1}+w_{0}=\widetilde{L}^{(1)} .
$$

In the framework of the equivalence class (104) of real self-adjoint operators we can always arrange that $w_{n}=$ const if $w_{n}=\exp f_{n} \neq 0$. However, these transformations realize a formal equivalence of the spectral theories only on the zero level $L \psi=0$, and therefore we can apply the Laplace transformation to all levels, generally speaking, only once (for special cases see below).

The proof of the lemma can be obtained by direct verification.

An investigation of the cyclic chains of difference operators has not been carried out. The classes of purely factorized operators of the form a) and b):

$$
\text { a) } L=Q^{+} Q ; \quad \text { b) } \widetilde{L}=Q Q^{+}
$$

are of interest, where

$$
Q=1+c e^{l_{1}(n)} T_{1}+d e^{l_{2}(n)} T_{2},
$$

and

$$
l_{j}(n)=l_{j 1} n_{1}+l_{j 2} n_{2}
$$

are linear forms in $n=\left(n_{1}, n_{2}\right)$.

Following [43], 44], we consider the 'equation of black triangles', which have the form $\left\langle n, n+T_{1}, n+T_{2}\right\rangle$ :

$$
Q \psi=0,
$$

and the 'equation of white triangles', which have the form $\left\langle n, n-T_{1}, n-T_{2}\right\rangle$ :

$$
Q^{+} \psi=0 .
$$

Theorem 4 1) The equation of black triangles $Q \psi=0$ certainly has an infinitedimensional space of solutions $\psi \in \mathcal{L}_{2}\left(\mathbb{Z}^{2}\right)$ if one of the following conditions is satisfied:

a) $l_{11}, l_{22}>0, l_{11} l_{22}-l_{12}^{2}>0$ 
b) $l_{11}, l_{22}>0, l_{11} l_{22}-l_{21}^{2}>0$;

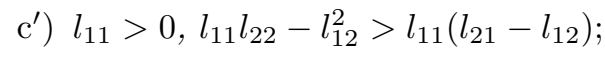

$\left.\mathrm{c}^{\prime \prime}\right) l_{22}>0, l_{11} l_{22}-l_{21}^{2}>l_{22}\left(l_{21}-l_{12}\right)$.

2) The equation of white triangles $Q^{+} \psi=0$ certainly has an infinite-dimensional space of solutions $\psi \in \mathcal{L}_{2}\left(\mathbb{Z}^{2}\right)$ if one of the following conditions, which are obtained from the previous ones by the transformation $l_{i j} \rightarrow-l_{i j}$, is satisfied:
a) $l_{11}, l_{22}<0, l_{11} l_{22}-l_{12}^{2}>0$;
b) $l_{11}, l_{22}<0, l_{11} l_{22}-l_{21}^{2}>0$;
c') $l_{11}<0, l_{11} l_{22}-l_{12}^{2}>l_{11}\left(l_{12}-l_{21}\right)$;
$\left.\mathrm{c}^{\prime \prime}\right) l_{22}<0, l_{11} l_{22}-l_{21}^{2}>l_{22}\left(l_{12}-l_{21}\right)$.

Proof We construct explicitly the solutions of our equations if the conditions presented above are satisfied. We examine first the case of black triangles. We are looking for a solution of the form

$$
\psi_{n}=e^{-K_{2}(n)} \chi_{n},
$$

where

$$
K_{2}(n)=\alpha n_{1}^{2}+2 \beta n_{1} n_{2}+\delta n_{2}^{2}
$$

For $\chi_{n}$ we obtain the equation

$$
0=\chi_{n}+c e^{-\alpha} e^{\left(l_{11}-2 \alpha\right) n_{1}+\left(l_{12}-2 \beta\right) n_{2}} \chi_{n+T_{1}}+d e^{-\delta} e^{\left(l_{21}-2 \beta\right) n_{1}+\left(l_{22}-2 \delta\right) n_{2}} \chi_{n+T_{2}} .
$$

We consider three cases:

a) the coefficients (106) depend only on $n_{1}$;

b) the coefficients (106) depend only on $n_{2}$;

c) the coefficients (106) depend only on $n_{1}+n_{2}$.

This leads, respectively, to the conditions:
a) $l_{12}=2 \beta, l_{22}=2 \delta$;
b) $l_{11}=2 \alpha, l_{21}=2 \beta$;
c) $l_{11}-2 \alpha=l_{12}-2 \beta, l_{21}-2 \beta=l_{22}-2 \delta$.

In the case a) we make the substitution

$$
\chi_{n}=w^{n_{2}} \varphi_{n_{1}}
$$

and obtain the equation for $\varphi_{m}$ :

$$
0=\varphi_{m}+c e^{-\alpha} e^{\left(l_{11}-2 \alpha\right)(m+1)} \varphi_{m+1}+w d e^{-\delta} e^{\left(l_{21}-l_{12}\right) m} \varphi_{m} .
$$


In addition we put $l_{11}=2 \alpha$. After this we obtain

$$
\left(1+w c e^{-l_{22} / 2} e^{\left(l_{21}-l_{12}\right) m}\right) \varphi_{m}=-c e^{-\alpha} \varphi_{m+1} .
$$

Two cases are possible.

Case 1. $l_{21}>l_{12}$. We choose the value of $w_{q}$ such that

$$
1+w_{q} d e^{-\delta / 2} e^{\left(l_{21}-2 \beta\right) q}=0
$$

where $2 \delta=l_{22}, 2 \beta=l_{12}$. ¿From this we obtain the 'quantization condition', which selects a discrete series of admissible values $w=w_{q}$, where $q$ runs over $\mathbb{Z}$. Under this condition $\varphi_{m}^{(q)}=0$ for $m>q$.

Further, we consider the relation

$$
\varphi_{m}^{(q)}=-\left(c e^{-\alpha}\right)\left(1+w_{q} e^{k_{2}-\delta} e^{\left(l_{21}-2 \beta\right) m}\right)^{-1} \varphi_{m+1}
$$

as $m \rightarrow \infty$. If $l_{21}-2 \beta>0$, then for $m<0$ and $|m| \rightarrow \infty$ we have the asymptotics

$$
\varphi_{m}^{(q)} \sim(\text { const })^{|m|} .
$$

Our solution is constructed in the form

$$
\psi_{n}^{(q)}=e^{-K_{2}(n)} w^{n_{2}} \varphi_{n_{1}}^{(q)},
$$

where $2 K_{2}(n)=l_{11} n_{1}^{2}+2 l_{12} n_{1} n_{2}+l_{22} n_{2}^{2}$. This form is strictly positive if and only if

$$
l_{11}>0, \quad l_{22}>0, \quad l_{11} l_{22}-l_{12}^{2}>0 .
$$

The function $\psi_{n}^{(q)}$ certainly belongs to the space $\mathcal{L}_{2}\left(\mathbb{Z}^{2}\right)$ and takes the value zero for $n_{1}>q$.

Case 2. $l_{12} \geq l_{21}$. In this case a solution $\varphi_{m}$ of equation (108) grows no faster than (const) ${ }^{|m|}, m \rightarrow \pm \infty$, for any $w$. Thus we have constructed the necessary solution for the case a) of black triangles.

In the case b) we interchange the position of $n_{1}$ and $n_{2}$. In the case c) we require that $\varphi$ depends only on $n_{1}+n_{2}$. For example, in case $\mathrm{c}^{\prime}$ ) we put

$$
2 K_{2}(n)=l_{11} n_{1}^{2}+2 l_{12} n_{1} n_{2}+\left(l_{22}-l_{21}+l_{12}\right) n_{2}^{2}
$$

and make the substitution

$$
\chi_{n}=w^{n_{1}} \varphi_{n_{1}+n_{2}} .
$$

For the function $\varphi_{n}$ we obtain the following equation:

$$
\varphi_{n}+\left(c w e^{-l_{11} / 2}+d e^{\left(l_{21}-l_{12}-l_{22}\right) / 2} e^{\left(l_{21}-l_{12}\right)\left(n_{1}+n_{2}\right)}\right) \varphi_{n+1}=0 .
$$

In the cases a)-c) for white triangles we proceed completely analogously.

Other cases, when the solutions lie in $\mathcal{L}_{2}\left(\mathbb{Z}^{2}\right)$, have not been found so far. The form $K_{2}(n)$ can be written as follows.

The case of black triangles: 

a) $2 K_{2}=\left(\begin{array}{ll}l_{11} & l_{12} \\ l_{12} & l_{22}\end{array}\right)$;
b) $2 K_{2}=\left(\begin{array}{ll}l_{11} & l_{21} \\ l_{21} & l_{22}\end{array}\right)$;
c') $2 K_{2}=\left(\begin{array}{cc}l_{11} & l_{12} \\ l_{12} & l_{22}-l_{21}+l_{12}\end{array}\right)$;
$\left.c^{\prime \prime}\right) 2 K_{2}=\left(\begin{array}{cc}l_{11}-l_{21}+l_{12} & l_{21} \\ l_{21} & l_{22}\end{array}\right)$.

The case of white triangles:
a) $2 K_{2}=\left(\begin{array}{ll}-l_{11} & -l_{12} \\ -l_{12} & -l_{22}\end{array}\right)$;
b) $2 K_{2}=\left(\begin{array}{ll}-l_{11} & -l_{21} \\ -l_{21} & -l_{22}\end{array}\right)$;
c') $2 K_{2}=\left(\begin{array}{cc}-l_{11} & -l_{12} \\ -l_{12} & -l_{22}+l_{21}-l_{12}\end{array}\right)$;
$\left.c^{\prime \prime}\right) 2 K_{2}=\left(\begin{array}{cc}-l_{11}+l_{21}-l_{12} & -l_{21} \\ -l_{21} & -l_{22}\end{array}\right)$.

Remark 11 We can impose analogous conditions on the coefficients $l_{i j}$ and their relation with $\alpha, \beta, \delta$, demanding that the coefficients of equation (106) for $\chi_{n}$ be dependent only on a combination of the form $\left(\kappa n_{1}+\tau n_{2}\right)$. If $\left(\kappa n_{1}+\tau n_{2}\right)$ and $\left(u n_{1}+v n_{2}\right)$ are the basis of the lattice, then the condition of dependency on only one variable has the form:

$$
\begin{aligned}
\left(l_{11}-2 \alpha\right) \tau & =\left(l_{12}-2 \beta\right) \kappa, \\
\left(l_{21}-2 \beta\right) \tau & =\left(l_{22}-2 \delta\right) \kappa .
\end{aligned}
$$

Now we can seek a solution in the form

$$
\chi_{n}=w^{u n_{1}+v n_{2}} \varphi_{\kappa n_{1}+\tau n_{2}} .
$$

Further, for the functions $\varphi_{s}, s \in \mathbb{Z}$, we obtain a difference equation of the form

$$
0=\varphi_{s}+A(s) \varphi_{s+\kappa}+B(s) \varphi_{s+\tau}
$$

Only in the three cases mentioned above can this equation be easily solved:
a) $\kappa=1, \tau=0$
b) $\kappa=0, \tau=1$;
c) $\kappa=\tau=1$.

In the other cases we obtain difference equations of order 2 and higher. We do not know how we could find solutions of them that lead to functions $\psi \in \mathcal{L}_{2}\left(\mathbb{Z}^{2}\right)$.

¿From the theorem we have the following corollary. 
Corollary 1 Under the conditions stated in the theorem the positive operators $L=Q Q^{+}$or $\widetilde{L}=Q^{+} Q$ have spectrum in $\mathcal{L}_{2}\left(\mathbb{Z}^{2}\right)$ such that the point $\lambda=0$ is an infinitely degenerate point of the discrete spectrum. The eigenfunctions of this point satisfy the equation of black or white triangles

$$
\text { 1) } Q \psi=0 \text { or 2) } Q^{+} \psi=0 \text {. }
$$

The eigenfunctions of the ground state $\lambda=0$ can be determined by explicit formulae, which follow from the construction given above.

This assertion is a difference analogue of results in [7], [8], [36] on the ground states of the Pauli operator in a continuous purely magnetic case.

Problems 1. Prove that the constructed spaces of solutions of the equations $Q \psi=0$ and $Q^{+} \psi=0$ are complete in $\mathcal{L}_{2}\left(\mathbb{Z}^{2}\right)$ among all solutions, and find appropriate orthonormal bases. Explain what kind of cases of solubility in $\mathcal{L}_{2}\left(\mathbb{Z}^{2}\right)$ of the equations of black or white triangles exist, apart from the solutions found above. Even in the class of operators whose coefficients are exponents of linear forms $l_{j}(n)$, this problem has not been solved.

2. Prove that if the conditions of the theorem are satisfied, then the spectrum of the operators $L=Q Q^{+}$and $\widetilde{L}=Q^{+} Q$ has a non-trivial gap $\Delta$ between the ground state $\lambda_{0}=0$ and the next level $\lambda_{1} \geq \Delta>0$.

3. Find the class of operators $Q$ such that the spectrum of the operators $L=Q Q^{+}$and $\widetilde{L}=Q^{+} Q$ begins at the point $\lambda=0$ and is continuous, that is, the point $\lambda=0$ is the bottom of the continuous spectrum $(\Delta=0)$.

The last problem is of special interest. Below we consider algebraic conditions of the type of commutation of the operators $Q, Q^{+}$, which supposedly lead to the situation discussed in Problem 3.

To begin with we consider the difference (purely real) $q$-analogues of the continuous Schrödinger-Landau operator in a homogeneous (constant) magnetic field.

Suppose that the operators $Q, Q^{+}$have the form (105):

$$
Q_{c, d}=1+c e^{l_{1}(n)} T_{1}+d e^{l_{2}(n)} T_{2},
$$

and depend on the parameters

$$
c=e^{k_{1}}, \quad d=e^{k_{2}}, \quad l_{j}=l_{j 1} n_{1}+l_{j 2} n_{2} .
$$

Lemma 166 Operators $Q, Q^{+}$of the form (105) satisfy the relation

$$
Q_{c, d} Q_{c, d}^{+}-1=q\left(Q_{c^{\prime}, d^{\prime}}^{+} Q_{c^{\prime}, d^{\prime}}-1\right)
$$

if the matrix $l_{i j}$ and the parameters $c, d, c^{\prime}, d^{\prime}$ are related by the following equalities:

$$
\begin{array}{cl}
c=u^{-2} c^{\prime}, \quad & d=u^{-2} d^{\prime}, \quad q=u^{-2}, \quad u=e^{l_{11}}, \\
& 2 l_{11}=2 l_{22}=l_{12}+l_{21} .
\end{array}
$$


Remark 12 In the notation

$$
e^{l_{11}}=u, \quad e^{l_{12}}=v
$$

the operator $Q$ is written in the form

$$
Q_{c, d}=1+c u^{n_{1}} v^{n_{2}} T_{1}+d\left(u^{2} / v\right)^{n_{1}} u^{n_{2}} T_{2} .
$$

Dependence on the parameters $(u, v)$ in (114) is omitted. Such notation was used in 43], where these operators were introduced for the first time and their eigenfunctions were found.

The proof of the lemma is by direct verification.

Comparing the results of Lemma 16 and Theorem 4, we obtain the following result $(43)$.

Theorem 5 We consider the operators $L=Q Q^{+}$and $\widetilde{L}=Q^{+} Q$ under the conditions (115). The spectrum of these operators in $\mathcal{L}_{2}(\mathbb{Z})$ for $0 \leq \lambda<1$ is purely discrete, infinitely degenerate, and can only lie at the following points:

$$
\begin{aligned}
& \text { a) } \lambda_{j}=1-u^{2 j}, j \geq 0, \quad u<1 \\
& \text { b) } \lambda_{j}=1-u^{-2 j}, j \geq 0, \quad u>1 .
\end{aligned}
$$

In the following cases the spectrum of the operator $L$ occupies all points (117), and the spectrum of the operator $\widetilde{L}$ occupies all points (117) except $\lambda_{0}$ :

$$
\left.\left.\mathrm{a}^{\prime}\right) \quad u^{-3}>v^{-1}>u^{-1}>1 ; \quad \mathrm{a}^{\prime \prime}\right) u^{-1}>\max \left(v, v^{-1}\right) \geq 1 .
$$

Analogously, the spectrum of the operator $\widetilde{L}$ occupies all points (118), and $L$ occupies all points except $\lambda_{0}$, if one of the following conditions is satisfied:

$$
\left.\left.\mathrm{b}^{\prime}\right) \quad u^{3}>v>u>1 ; \quad \mathrm{b}^{\prime \prime}\right) \quad u>\max \left(v, v^{-1}\right) \geq 1 .
$$

All eigenfunctions discussed in the theorem are obtained from solutions of the equations $Q \psi=0$ or $Q^{+} \psi=0$ for proper values of the constants $c, d, u, v$ by the use of 'creation operators':

1) $Q_{c, d} \widetilde{\psi}_{0}=0, \widetilde{L}_{c, d} \widetilde{\psi}_{0}=Q_{c_{0}, d_{0}}^{+} Q_{c_{0}, d_{0}} \widetilde{\psi}_{0}=0, \widetilde{\psi}=Q_{c_{0}, d_{0}}^{+} \circ \cdots \circ Q_{c_{k-1}, d_{k-1}}^{+} \widetilde{\psi}_{0}$, $\widetilde{L}_{c_{0}, d_{0}} \psi=\left(1-u^{ \pm 2 k}\right) \psi$;

2) $Q^{+} \psi_{0}=0, L_{c, d} \psi_{0}=Q_{c, d} Q_{c, d}^{+} \psi_{0}=0, \psi=Q_{c_{0}, d_{0}} \circ \cdots \circ Q_{c_{k-1}, d_{k-1}} \psi_{0}$, $L_{c_{0}, d_{0}} \psi=\left(1-u^{ \pm 2 k}\right) \psi$,

$$
c_{j}=u^{2} c_{j-1}, \quad d_{j}=u^{2} d_{j-1} .
$$

Remark 13 Of the two cases mentioned in the proof of Theorem 4, in the given situation only case 1 is realized, in which the eigenfunctions $\psi^{(q)}$ that we found vanish on the whole subplane. 
The situation described in Theorem 5 is completely analogous to 22], that is, to Theorem 1 in $\S 3$. The difference is that for $n=2$ the spaces of solutions are infinite-dimensional (the completeness of the solutions found on the corresponding levels has not been established so far).

We note that conditions (119), (120) are obtained directly from the condition of Theorem 4, but the case c) here is not realized under conditions (115).

Problem Prove that the spectrum of the operators $L=Q_{c, d} Q_{c, d}^{+}$and $\widetilde{L}=$ $Q_{c, d}^{+} Q_{c, d}$ in the space $\mathcal{L}_{2}\left(\mathbb{Z}^{2}\right)$ for $\lambda \geq 1$ is continuous and runs over the whole semi-axis $\lambda \geq 1$. As we assume, this is a simple Lebesgue spectrum.

We point out that our operators $Q$ depend on the constants $c, d, u, v$ (if the dependence on $u, v$ is not written explicitly, then in the given formula we assume that $u, v$ have not changed). The following formulae are true:

$$
\begin{array}{lll}
T_{1} Q_{c, d} T_{1}^{-1}=Q_{c^{\prime}, d^{\prime}}, & c^{\prime}=c d^{l_{11}}, & d^{\prime}=d e^{l_{12}}, \\
T_{2} Q_{c, d}^{+} T_{2}^{-1}=Q_{c^{\prime \prime}, d^{\prime \prime}}, & c^{\prime \prime}=c d^{l_{12}}, & d^{\prime \prime}=d e^{l_{22}},
\end{array}
$$

where

$$
\begin{aligned}
& Q_{c, d}=1+c e^{l_{1}(n)} T_{1}+d e^{l_{2}(n)} T_{2}, \\
& Q_{c, d}^{+}=q+c e^{l_{1}\left(n-T_{1}\right)} T_{1}^{-1}+d e^{l_{2}\left(n-T_{2}\right)} T_{2}^{-1} .
\end{aligned}
$$

The same is also true for the operators $L=Q Q^{+}, \widetilde{L}=Q^{+} Q$ :

$$
\begin{aligned}
& T_{1} L_{c, d} T_{1}^{-1}=L_{c^{\prime}, d^{\prime}}, \\
& T_{2} L_{c, d} T_{2}^{-1}=L_{c^{\prime \prime}, d^{\prime \prime}} .
\end{aligned}
$$

Definition 11 A characteristic vector-section is a function $\psi_{c, d}(n)$ that is analytically dependent on all the parameters $c, d, u, v$ and such that

$$
L \psi=\lambda \psi \quad \text { or } \quad \widetilde{L} \psi=\lambda \psi,
$$

where $\lambda$ does not depend on the parameters. A Bloch vector-section $\psi_{c, d}(n, p)$ is a function $\psi$ such that

$$
L \psi=\lambda \psi, \quad T_{1} \psi_{c, d}=e^{i p_{1}} \psi_{c^{\prime}, d^{\prime}}, \quad T_{2} \psi_{c, d}=e^{i p_{2}} \psi_{c^{\prime \prime}, d^{\prime \prime}} .
$$

By (121) the functions $T_{j} \psi_{c, d}$ satisfy these equations, but with shifted parameters $\left(c^{\prime}, d^{\prime}\right)$ or $\left(c^{\prime \prime}, d^{\prime \prime}\right)$. This definition makes sense also in the one-dimensional case (54), where we had

$$
\begin{array}{r}
Q_{c}=1+c e^{l(n)} T, \quad l(n)=l n, \\
T=T_{1}, \quad n=n_{1}, \quad T_{1} Q_{c} T_{1}^{-1}=Q_{c a}, \quad a=e^{l} .
\end{array}
$$

Let us construct the Bloch vector-section for $\lambda=0$ when $a>1$. We choose $c=c_{0}>0$. Choosing an arbitrary $\psi_{0, c_{0}}$, we put

$$
\psi_{c_{0} a^{m}}(n, p)=T_{1}^{m}\left(\psi_{0, c_{0}}(n)\right) e^{-i m p} .
$$


Limitations on the construction appear as $a \rightarrow 1$. There are no solutions at all in $\mathcal{L}_{2}(\mathbb{Z})$ for $a<1$ if we are talking about the equation $Q_{c} \psi=0$ corresponding to $\lambda=0$. Therefore, $a \geq 1, c \neq 0$.

As $a \rightarrow 1+0$ the operator tends to an operator with constant coefficients, where there are singularities of the Bloch vector-section.

Apparently, as $|c| \rightarrow 1, a \rightarrow 1$ we can talk about the transition of the Bloch vector-section corresponding to $\lambda \leq 1$ into an ordinary Bloch solution for the equation with constant coefficients (that is, a solution of pure exponent type), since for $c \neq 0$ the spectrum of the operator for $a=1$ has the form $(1-|c|)^{2} \leq$ $\lambda \leq(1+|c|)^{2}$.

In addition, for $a=1$ the spectrum is bounded in $\mathcal{L}_{2},|\lambda| \leq(1+|c|)^{2}$, and for $a>1$ the operator is of course unbounded. Hence, we necessarily have a singularity here. For $\lambda \geq 1$ the situation is not clear.

Problem For the operators $L=Q Q^{+}$or $\widetilde{L}=Q^{+} Q$, whose coefficients are exponents of linear functions, construct a complete basis of eigenfunctions in the form of 'Bloch sections' that depend analytically on all the parameters $c, d, l_{i j}$, which has minimally possible singularities in the submanifolds, where we have operators with constant coefficients in one of the variables.

We return now to the case when the point $\lambda=0$ is supposedly the lower bound of the continuous spectrum for operators of the form

$$
L=Q Q^{+}
$$

where the $Q$ have the form (105).

Hypothesis If the operators $Q, Q^{+}$commute, $Q Q^{+}=Q^{+} Q$, and they have a general solution $Q \psi=0, Q^{+} \psi=0$ that is bounded on the lattice $\mathbb{Z}^{2}$ (or is growing sufficiently slowly?), then the point $\lambda=0$ is the lower bound of the continuous spectrum in $\mathcal{L}_{2}\left(\mathbb{Z}^{2}\right)$ for the operator $L=\widetilde{L}$. We state below a natural generalization of this hypothesis.

Example 10 Let the operators $Q_{c, d}, Q_{c, d}^{+}$be such that $l_{11}=l_{22}=0$ or $u=1$. Then we have

$$
Q_{c, d} Q_{c, d}^{+}=Q_{c, d}^{+} Q_{c, d},
$$

as follows from (114). The operators have the form

$$
\begin{aligned}
& Q_{c, d}=1+c v^{n_{2}} T_{1}+d v^{-n_{1}} T_{2}, \\
& Q_{c, d}^{+}=1+c v^{n_{2}} T_{1}^{-1}+d v^{-n_{1}} T_{2}^{-1} .
\end{aligned}
$$

We pose the following question. Let two operators be given in the form

$$
Q_{1}=1+a_{n} T_{1}+b_{n} T_{2}, \quad Q_{2}=1+c_{n} T_{1}^{-1}+d_{n} T_{2}^{-1}
$$


with non-vanishing coefficients. We consider the system of equations of black and white triangles simultaneously:

$$
\left\{\begin{array}{l}
Q_{1} \psi=0, \\
Q_{2} \psi=0 .
\end{array}\right.
$$

In this case is the system (127) completely locally consistent?

Richer in content is the following formulation. Let the 'initial condition' for (127) be given in the form of two arbitrary values of $\psi$ at the ends of any edge of the lattice (for example, $\psi_{n-T_{1}}$ and $\psi_{n}$ ). Using equations (127), we can solve the system and find the value at any other point $n^{\prime} \in \mathbb{Z}^{2}$, moving along the paths of the triangles, black and white: knowing the values at the ends of any edge, we find the value at the third vertex of any black or white triangle that has this edge as its border. Considering a bundle of six triangles with a common vertex $n$, we can pass from one cycle and return again to the edge $\left[n-T_{1}, n\right]$, and the linear 'transformation of curvature' will be given by an upper triangular $(2 \times 2)$-matrix:

$$
\begin{array}{r}
\psi_{n-T_{1}}^{(\text {new })}=A_{n} \psi_{n-T_{1}}^{(\text {old })}+B_{n} \psi_{n}^{(\text {old })}, \\
\psi_{n}^{(\text {new })}=\psi_{n}^{(\text {old })} .
\end{array}
$$

The matrix coefficients are the 'curvatures' $A_{n}, B_{n}$. They are expressed in terms of the coefficients of the system (127):

$$
\begin{aligned}
A_{n} & =\frac{b_{n-T_{1}} d_{n+T_{1}}}{b_{n} c_{n+T_{2}} d_{n} a_{n-T_{2}}} \\
B_{n} & =\frac{b_{n-T_{1}}}{c_{n+T_{2}}}\left(\frac{1}{b_{n}}\left(\frac{d_{n+T_{1}}}{a_{n-T_{2}}}\left(\frac{1}{d_{n}}-b_{n-T_{2}}\right)-c_{n+T_{1}}+1\right)-d_{n+T_{2}}\right)-a_{n-T_{1}} .
\end{aligned}
$$

Lemma 177 The condition $A_{n}=1, B_{n}=0$ for all $n \in \mathbb{Z}^{2}$ is necessary and sufficient for the possibility of a unique solution of the system (127) on the plane $\mathbb{Z}^{2}$ under arbitrary initial conditions imposed at the ends of any fixed edge. For this it is also necessary and sufficient to satisfy the following algebraic relation for the operators $Q_{1}, Q_{2}$ :

$$
\left(\left(Q_{1}-1\right)\left(Q_{2}-1\right)-1\right)=f_{n}\left(\left(Q_{2}-1\right)\left(Q_{1}-1\right)-1\right), \quad f_{n} \neq 0 .
$$

We omit the proof of the lemma.

Corollary 2 If the system (127) is completely consistent, then its solution is determined by an arbitrary solution of a one-dimensional difference equation of the second order along any path of edges without self-crossing, in particular, along the path $n_{1}=$ const (we obtain an equation for the variable $n_{2}$ ) or $n_{2}=$ const (we obtain an equation for the variable $n_{1}$ ). If the curvature is trivial, then all these equations are equivalent. 
Example 11 For the commuting operators $Q_{1}=Q_{2}^{+}=Q_{c, d}$ from (125) the condition for consistency is satisfied. For the variable $n_{1}\left(n_{2}=\right.$ const $)$ we get the equation

$$
c v^{n_{2}}\left(\psi_{n-T_{1}}+\psi_{n+T_{1}}\right)+\left(1+c^{2} v^{2 n_{2}}-d v^{-2 n_{1}}\right) \psi_{n}=0 .
$$

To end this section we give some information on factorizations and Laplace transformations for general non-self-adjoint operators $L$ of the second order on an equilateral triangular lattice, and also on complex Hermite operators. The following assertions hold.

Theorem 6 To represent the operator

$$
L=a_{n}+b_{n} T_{1}+c_{n} T_{2}+d_{n-T_{1}} T_{1}^{-1} T_{2}+e_{n-T_{1}} T_{1}^{-1}+f_{n-T_{2}} T_{2}^{-1}+g_{n-T_{2}} T_{1} T_{2}^{-1}
$$

in the form
a) $L=Q_{1} Q_{2}+w_{n}$ or
b) $L=Q_{2} Q_{1}+w_{n}$,

where $Q_{1}$ and $Q_{2}$ have the form

$$
Q_{1}=x_{n}+y_{n} T_{1}+z_{n} T_{2}, \quad Q_{2}=p_{n}+q_{n-T_{1}} T_{1}^{-1}+r_{n-T_{2}} T_{2}^{-1},
$$

it is necessary and sufficient to satisfy the conditions

$$
\begin{aligned}
& \text { a) } b_{n+T_{2}} d_{n} f_{n+T_{1}}=g_{n} e_{n+T_{2}} c_{n+T_{1}} \text {, } \\
& \text { b) } f_{n} d_{n} b_{n}=c_{n} e_{n} g_{n} .
\end{aligned}
$$

If both conditions are satisfied, then the Laplace transformations

$$
\begin{aligned}
& \text { a) } L=Q_{1} Q_{2}+w \longmapsto \widetilde{L}=Q_{2} w^{-1} Q_{1}+1, \\
& \text { b) } L=Q_{2} Q_{1}+v \longmapsto \widetilde{L}=Q_{1} w^{-1} Q_{2}+1
\end{aligned}
$$

can be iterated, moreover infinitely many times (if $w, v \neq 0$ ).

Remark 14 The factorization conditions (132) are invariant with respect to the rotation of the lattice by the angle $2 \pi / 3$. By analogy with Definition 10 we can introduce six types of Laplace transformations. Then the 1st, 3rd and 5th transformations are unitarily adjoint and inverse to the 4th, 6th and 2nd transformations respectively. The same applies to the following assertion.

Theorem 7 To factorize an operator $L$ of the form

$$
L=a_{n}+b_{n} T_{1}+\bar{c}_{n} T_{2}+d_{n-T_{1}} T_{2} T_{1}^{-1}+\bar{b}_{n-T_{1}} T_{1}^{-1}+c_{n-T_{2}} T_{2}^{-1}+\bar{d}_{n-T_{2}} T_{1} T_{2}^{-1}
$$

in the form
a) $L=Q Q^{+}+w_{n} \quad$ or
b) $L=Q^{+} Q+w_{n}$, 
it is necessary and sufficient to satisfy the following conditions:

$$
\begin{aligned}
& \text { a) } b_{n-T_{1}} c_{n-T_{2}} d_{n-T_{1}-T_{2}} \in \mathbb{R} \text {, } \\
& \text { b) } d_{n} c_{n} b_{n} \in \mathbb{R} .
\end{aligned}
$$

In particular, if both conditions are satisfied, then the Laplace transformations can be iterated infinitely many times.

The proof of these theorems is straightforward.

For complex Hermite operators $L$ it is natural to define a class of 'phase' gauge transformations

$$
L \mapsto e^{i f_{n}} L e^{-i f_{n}}, \quad \psi_{n} \mapsto e^{i f_{n}} \psi_{n}
$$

such that $f_{n} \in \mathbb{R}$. These transformations keep the operator formally Hermitian with respect to the previous standard scalar product in $\mathcal{L}_{2}\left(\mathbb{Z}^{2}\right)$

$$
\langle\varphi, \psi\rangle=\sum_{n \in \mathbb{Z}^{2}} \varphi_{n} \bar{\psi}_{n} .
$$

The operator $L$ naturally has 'real' and 'phase' projections

$$
\begin{aligned}
& L_{\mathbb{R}}=a_{n}+\beta_{n} T_{1}+\gamma_{n} T_{2}+\delta_{n-T_{1}} T_{2} T_{1}^{-1}+\beta_{n-T_{1}} T_{1}^{-1}+\gamma_{n-T_{2}} T_{2}^{-1}+\delta_{n-T_{2}} T_{1} T_{2}^{-1}, \\
& L_{\Phi}=a_{n}+B_{n} T_{1}+\bar{C}_{n} T_{2}+D_{n-T_{1}} T_{2} T_{1}^{-1}+\bar{B}_{n-T_{1}} T_{1}^{-1}+C_{n-T_{2}} T_{2}^{-1}+\bar{D}_{n-T_{2}} T_{1} T_{2}^{-1},
\end{aligned}
$$

$b_{n}=\beta_{n} B_{n}, c_{n}=\gamma_{n} C_{n}, d_{n}=\delta_{n} D_{n}$, where $\beta_{n}, \gamma_{n}, \delta_{n}, a_{n}, w_{n}$ are real numbers and $B_{n}, C_{n}, D_{n}$ are complex with modulus equal to 1 .

Definition 12 A physical magnetic field is the phase part of the product of the coefficients of an operator (of connection) along the border of any black or white triangle

$$
\begin{array}{rlr}
e^{-i \Phi_{n}^{(1)}}=B_{n-T_{1}} C_{n-T_{2}} D_{n-T_{1}-T_{2}}, & \text { (black) } \\
e^{i \Phi_{n}^{(2)}}=D_{n} C_{n} B_{n} . & \text { (white) }
\end{array}
$$

The following lemma is obvious.

Lemma 188 The operator $L$ is reduced by the phase gauge transformation $e^{i f_{n}} L e^{-i f_{n}}$ to a purely real form if and only if the magnetic field (that is, all 'magnetic fluxes' $\left.\Phi_{n}^{(j)}\right)$ is trivial:

$$
\Phi_{n}^{(j)}=0, \quad n \in \mathbb{Z}^{2}, \quad j=1,2 .
$$

Corollary 3 A complex Hermite operator $L$ admits an unbounded number of Laplace transformations of all six forms if and only if it is reduced by a phase gauge transformation to a purely real operator. 
Thus, the $q$-analogues of the Schrödinger-Landau operator on the lattice have no connection with the magnetic field. Difference operators in a physical magnetic field do not factorize, generally speaking. A proper analogue of an operator in a homogeneous magnetic field can (having chosen a specific gauge transformation, that is, a 'vector-potential' composed of coefficients $b_{n}, c_{n}, d_{n}$ such that $\left|b_{n}\right|=\left|c_{n}\right|=\left|d_{n}\right|=1$ ) be written in the form

$$
\begin{gathered}
L=6-e^{i \Phi n_{2}} T_{1}-e^{-i \Phi n_{1}} T_{2}-e^{i \Phi\left(n_{1}+n_{2}\right)} T_{2} T_{1}^{-1} \\
-e^{-i \Phi n_{2}} T_{1}^{-1}-e^{i \Phi n_{1}} T_{2}^{-1}-e^{-i \Phi\left(n_{1}+n_{2}\right)} T_{1} T_{2}^{-1} .
\end{gathered}
$$

\section{§7. Factorizations and Laplace transformations on many-dimensional lattices of regular tetahedra in $\mathbb{R}^{N}$}

We consider a lattice in $\mathbb{R}^{N}$ such that the ends of the basis vectors $T_{1}, \ldots, T_{N}$ of the lattice together with the point 0 form a regular $N$-dimensional simplex (a tetrahedron for $N=3$ ). We call this simplex, and also all others obtained from it through translations by a vector of the lattice, black tetrahedra. White tetrahedra ( $N$-dimensional simplexes) are the tetrahedron $\left\langle 0,-T_{1}, \ldots,-T_{N}\right\rangle$ and all others obtained from it through integer-valued translations.

Two black (white) tetrahedra can have no more than one vertex in common. A black and a white tetrahedron can have the longest edge in common. This is true for any dimension $\geq 2$.

We consider a real self-adjoint operator of the form

$$
L=a_{n}+\sum_{k=1}^{N}\left(b_{k, n} T_{k}+b_{k, n-T_{k}} T_{k}^{-1}\right)+\sum_{k \neq j}\left(c_{k j, n-T_{j}} T_{k} T_{j}^{-1}+c_{k j, n-T_{k}} T_{j} T_{k}^{-1}\right) .
$$

We assume that the coefficients $b_{k, n}, c_{k j, n}$ are a real connection, and they are all non-zero.

Theorem 8 The operator $L$ admits a representation in the form

$$
L=Q Q^{+}+w_{n}
$$

where

$$
Q=x_{n}+\sum_{k=1}^{N} y_{k, n} T_{k},
$$

if and only if the following condition for the coefficients $b_{k, n}, c_{k j, n}$ is satisfied:

$$
\frac{b_{k, n-T_{k}} b_{j, n-T_{j}}}{c_{k j, n-T_{k}-T_{j}}}=x_{n} \text { does not depend on } k, j .
$$

Analogously, the condition for factorization $L=Q^{+} Q+w_{n}$ is the independence of the expression $b_{k, n} b_{j, n} / c_{k j, n}$ on $k, j$. 
Remark 15 For $N=2$ we do not have any conditions. For $N=3$ we have two conditions for any white tetrahedron: the product of the coefficients of connection that sit on pairs of opposite (skew) edges of the tetrahedron is the same. In all, the tetrahedron has six edges, which make up three skew pairs, hence we have two conditions. If we want to have all forms of factorizations, then this condition should also be satisfied for black tetrahedra.

The proof of the theorem, as before, is by direct verification.

We now consider factored operators, for which the coefficients of the corresponding operators $Q, Q^{+}$have the form of exponents of linear functions, as for $N=2$ :

$$
\begin{gathered}
Q=1+c e^{l_{1}(n)} T_{1}+d e^{l_{2}(n)} T_{2}+f e^{l_{3}(n)} T_{3}, \\
l_{j}(n)=\sum_{i=1}^{N} l_{j i} n_{i} .
\end{gathered}
$$

For such operators the following results are true.

Theorem 9 The equations

1) $Q \psi=0$ (the equation of black triangles)

or

$$
\text { 2) } Q^{+} \psi=0 \text { (the equation of white triangles) }
$$

certainly have infinite-dimensional spaces of solutions $\psi \in \mathcal{L}_{2}\left(\mathbb{Z}^{N}\right)$ if the following conditions are satisfied:

1)

$$
\begin{gathered}
\text { 1a) }\left(\begin{array}{lll}
l_{11} & l_{12} & l_{13} \\
l_{12} & l_{22} & l_{23} \\
l_{13} & l_{23} & l_{33}
\end{array}\right)>0, l_{32}=l_{23} \\
\text { 1b) }\left(\begin{array}{ccc}
l_{11} & l_{12} & l_{13} \\
l_{12} & l_{22}-l_{21}+l_{12} & l_{23} \\
l_{13} & l_{23} & l_{33}
\end{array}\right)>0, l_{23}+l_{31}=l_{32}+l_{13}
\end{gathered}
$$

2) the same with $l_{i j} \rightarrow-l_{i j}$;

and also the conditions obtained from those stated above by an arbitrary permutation of the indices $1,2,3$.

Corollary 4 If the conditions of the theorem are satisfied, the operators $L=$ $Q Q^{+}$and $\widetilde{L}=Q^{+} Q$ have $\lambda=0$ as the point of the discrete spectrum that is infinitely degenerate. 
Problem Prove that the eigenfunctions constructed according to the scheme of the proof of Theorem 4 give a complete basis for $\lambda=0$. Prove that the remaining spectrum is separated from $\lambda=0$ by a finite gap $\Delta>0$ in the space $\mathcal{L}_{2}\left(\mathbb{Z}^{3}\right)$.

Theorem 100 If the relations

$$
l_{i j}+l_{j i}=h
$$

are satisfied, then the operators $Q, Q^{+}$satisfy the relations

$$
Q_{c, d, f} Q_{c, d, f}^{+}-1=q\left(Q_{c^{\prime}, d^{\prime}, f^{\prime}}^{+} Q_{c^{\prime}, d^{\prime}, f^{\prime}}-1\right),
$$

where

$$
q=e^{h}, \quad c^{\prime}=e^{-h} c, \quad d^{\prime}=e^{-h} d, \quad f^{\prime}=e^{-h} f .
$$

This theorem generalizes Lemma 16, formulated for $N=2$.

It is essential to note that the conditions (146) contradict the condition of Theorem 9, and we cannot explicitly find solutions of the equations

$$
\begin{aligned}
& Q_{c, d, f} \psi=0, \\
& Q_{c, d, f}^{+} \psi=0,
\end{aligned}
$$

that belong to $\mathcal{L}_{2}\left(\mathbb{Z}^{2}\right)$ and are necessary to construct the spectrum of the operators $L, \widetilde{L}$.

Remark 16 As before, we can seek solutions of (141) in the form

$$
\psi_{n}=e^{-K_{2}(n)} \chi_{n},
$$

where $K_{2}(n)$ is chosen in such a way that the coefficients of the equation for $\chi_{n}$ do not contain the variable $n_{1}$. However, we have not succeeded in reducing the resulting equation to one variable. After the substitution

$$
\chi_{n}=w^{n_{1}} \varphi_{n_{2}, n_{3}}
$$

we arrive at the two-dimensional equation of triangles

$$
A_{n} \varphi_{n}+B_{n} \varphi_{n+T_{2}}+C_{n} \varphi_{n+T_{3}}=0 .
$$

Unfortunately, the coefficients of this equation are very complicated, and so far we do not know how to find solutions of it explicitly.

Obviously, one equation (141) can always be solved in the half-space of a definite direction if the initial conditions are imposed arbitrarily on vertices in any plane that has one of the four forms:

$$
n_{1}=\text { const, or } n_{2}=\text { const, or } n_{3}=\text { const, or } n_{1}+n_{2}+n_{3}=\text { const. }
$$


Here the equation of a tetrahedron (as of a triangle for $N=2$ ) is treated as the evolution equation. It is not reversible, as it is for $N=2$, since a solution in the inverse direction of 'time' is no longer local; its solubility depends on the initial condition belonging to a special functional class.

Now we consider the condition for consistency of the pair of equations (black and white)

$$
\begin{array}{ll}
Q_{1} \psi=0, & Q_{1}=1+x_{n} T_{1}+y_{n} T_{2}+z_{n} T_{3}, \\
Q_{2} \psi=0, & Q_{2}=1+p_{n} T_{1}^{-1}+q_{n} T_{2}^{-1}+r_{n} T_{3}^{-1} .
\end{array}
$$

Theorem 111 The system (152) is completely consistent, that is, its solution is determined by an arbitrary solution of some difference equation of the second order in one of the planes (151), if the relation

$$
\left(1-Q_{1}\right)\left(1-Q_{2}\right)-1=f_{n}\left(\left(1-Q_{2}\right)\left(1-Q_{1}\right)-1\right)
$$

is satisfied, where $f_{n}$ is a non-zero function (in particular, for $f_{n} \equiv 1$ this equality is transformed into the commutation condition $\left.Q_{1} Q_{2}=Q_{2} Q_{1}\right)$. The corresponding equation of the second order, for example for the plane $n_{1}+n_{2}+$ $n_{3}=$ const, has the form

$$
\left(1-Q_{1}\right)\left(1-Q_{2}\right) \psi=\psi
$$

Proof Since equations (152) have the form

$$
\left(1-Q_{1}\right) \psi=\psi, \quad\left(1-Q_{2}\right) \psi=\psi,
$$

we see that the conditions

$$
\left(1-Q_{1}\right)\left(1-Q_{2}\right) \psi=\psi
$$

and

$$
\left(1-Q_{2}\right)\left(1-Q_{1}\right) \psi=\psi,
$$

which are equations of the second order in the plane $n_{1}+n_{2}+n_{3}=$ const, should be equivalent. Hence it follows that there is a non-zero function $f_{n}$ such that

$$
\left(1-Q_{1}\right)\left(1-Q_{2}\right)-1=f_{n}\left(\left(1-Q_{2}\right)\left(1-Q_{1}\right)-1\right) .
$$

Thus, we have proved the theorem for the plane $n_{1}+n_{2}+n_{3}=$ const.

Writing our conditions locally, in the star of any vertex, we see that it is invariant with respect to rotations that transform the lattice into itself. All four directions of the planes mentioned above are equivalent. We have proved Theorem 11. 
Example 12 Let us consider the operators (147), where $h=0$. In this case the operators $Q, Q^{+}$commute. The corresponding equation in the hyperplane $n_{3}=$ const has the form

$$
\begin{array}{r}
0=\left(A+C^{2} e^{2 \alpha n_{2}}+D^{2} e^{-2 \alpha n_{1}}\right) \psi_{n}+C e^{\alpha n_{2}}\left(\psi_{n+T_{1}}+\psi_{n-T_{1}}\right) \\
+D e^{-\alpha n_{1}}\left(\psi_{n+T_{2}}+\psi_{n-T_{2}}\right)+C D e^{\alpha\left(n_{2}-n_{1}\right)}\left(e^{\alpha} \psi_{n+T_{2}-T_{1}}+e^{-\alpha} \psi_{n+T_{1}-T_{2}}\right) .
\end{array}
$$

Now we consider a 'vector factorization' for $N=3$, where the operators $Q^{+}$ represent vectors $\left(Q^{+\alpha}\right), \alpha=1,2$. Hence, the factorization of the operator is given in the form
a) WW $L=\sum_{\alpha=1}^{2} Q^{\alpha} Q^{+\alpha}+w_{n}$
b) $\mathrm{BB} L=\sum_{\alpha=1}^{2} Q^{+\alpha} Q^{\alpha}+w_{n}$,
c) $\mathrm{BW} L=Q^{1} Q^{+1}+Q^{+2} Q^{2}+w_{n}$,

where

$$
Q^{\alpha}=x_{n}^{\alpha}+y_{n}^{\alpha} T_{1}+z_{n}^{\alpha} T_{2}+t_{n}^{\alpha} T_{3} .
$$

Lemma 199 Vector factorization of a self-adjoint operator $L$ is always possible (although it is not unique). Moreover, we can regard the potential as a constant $w_{n}=w_{0}$.

The vector factorizations of all three forms generate Laplace transformations which, however, cannot be iterated.

The search for eigenfunctions of the ground state can sometimes be carried out in one of them:
a) $Q^{+1} \psi=0, Q^{+2} \psi=0$ for (WW),
b) $Q^{1} \psi=0, Q^{2} \psi=0$ for (BB),
c) $Q^{+1} \psi=0, Q^{2} \psi=0$ for (BW).

If the constant $w_{0}$ for the factorization is chosen correctly, then the lowest level is obtained from equations (156), a), b), or c).

It is difficult to find a criterion for the existence of one solution for the systems (156). However, as before, we state here a criterion for complete local consistency of these systems.

Theorem 122 The system (156) a) (or b) has solutions that are uniquely determined by arbitrary initial conditions imposed on the vertices of any straight line with direction vector $T_{1}, T_{2}, T_{3}$ or $T_{i} T_{j}^{-1}, i \neq j$, inside the dihedral angle in which lie the white (respectively, black) tetrahedra adjoining this straight line 
along an edge, if and only if the following condition is satisfied: for any four white (respectively, black) tetahedra that are located in angles of a tetrahedron twice the size, the eight equations corresponding to them are linearly dependent.

Proof For definiteness we consider the system (156) b). For the values of $\psi$ at the vertices of every black tetrahedron we have two equations. This means that if we know the values of $\psi$ at two vertices of a black tetrahedron, we can find the values at the other two. Hence we can easily see that if $\psi$ is given on all the vertices of some straight line, then our system dictates the extension of $\psi$ into the interior of the stated sector. Thus, we only need to clarify when this extension is possible for any initial conditions.

We consider a tetrahedron $T_{N}$, similar to the black one, with edge of length $N$. Let $V(N)$ be the number of black tetrahedra that lie in $T_{N}, V(N)=N(N+$ $1)(N+2) / 6$. For the values of $\psi$ at the $V(N+1)$ vertices that lie inside and on the border of $T_{N}$ we have $2 V(N)$ equations, of which only $V(N+1)-N-1$ should be linearly independent in order to satisfy the condition for complete consistency. But $2 V(N)-V(N+1)+N+1=V(N-1)$ is the number of double-size tetrahedra $T_{2} \subset T_{N}$.

The condition for consistency of the system (156) c) was considered in Theorem 11.

\section{$\S 8$. Factorizations of operators and Laplace trans- formations on two-dimensional surfaces}

Let us consider a two-dimensional manifold without boundary, triangulated in such a way that two-dimensional simplexes can be painted in two colours (black and white) so that two triangles that are adjacent along an edge have different colours. In this case an even number of triangles should meet at each vertex.

The metric on the surface is chosen in such a way that all triangles are equivalent to an equilateral triangle in the Euclidean plane. Hence it follows immediately that at any vertex the total curvature has the form $\left(2 \pi-N_{\mathrm{tr}} \pi / 3\right)$, where $N_{\mathrm{tr}}$ is the number of triangles meeting at a given vertex. If $N_{\mathrm{tr}}=6$, then the curvature is equal to zero. If $N_{\mathrm{tr}}=4$, then the curvature is positive. If $N_{\mathrm{tr}}>6$, then the curvature is negative.

Definition 13 A vertex scalar Schrödinger operator is an operator $L$ that acts on a function from a vertex according to the formula

$$
(L \psi)_{P}=\sum_{P^{\prime}} b_{P: P^{\prime}} \psi_{P^{\prime}}+a_{P} \psi_{P},
$$

where summation goes over the vertices $P^{\prime}$ that are nearest to $P$ but do not coincide with $P$. The condition that the operator $L$ is self-adjoint has the form

$$
b_{P^{\prime}: P}=\bar{b}_{P: P^{\prime}}, \quad a_{P} \in \mathbb{R} .
$$


Definition 14 1) A triangular (black) Schrödinger operator of type I is an operator $L$ that acts on functions $\psi_{T}$ of the black triangles $T$ in such a way that

$$
(L \psi)_{T}=\sum_{T^{\prime}} b_{T: T^{\prime}} \psi_{T^{\prime}}+a_{T} \psi_{T},
$$

where $T^{\prime}$ are all the black triangles that have a common vertex with $T$.

2) A triangular (black) Schrödinger operator of type II is an operator $L$ that acts on $\psi_{T}$ according to formula (159), but now $T^{\prime}$ runs over all black triangles that have with $T$ a common neighbouring (that is, adjacent along an edge) white triangle.

Analogously we define white triangular operators of types I and II. In both cases the self-adjointness condition with respect to the standard scalar product

$$
\langle\varphi, \psi\rangle=\sum_{T} \varphi_{T} \bar{\psi}_{T}
$$

has the form

$$
b_{T^{\prime}: T}=\bar{b}_{T: T^{\prime}}, \quad a_{T} \in \mathbb{R} .
$$

Theorem 133 Any real self-adjoint vertex operator $L$ admits a unique factorization of the form

$$
L=Q Q^{+}+w_{P}
$$

where

$$
\left(Q^{+} \psi\right)_{T}=\sum_{P} y_{T: P} \psi_{P}+x_{T} \psi_{T},
$$

and the sum is taken over all vertices $P$ of the black triangle T. For any black triangle $T$ and two of its vertices $P_{1}, P_{2}$ we have the equality (compare with (101)):

$$
y_{T: P_{1}} y_{T: P_{2}}=b_{P_{1}: P_{2}} .
$$

We have also an analogous assertion for white triangles.

Theorem 144 A real triangular black self-adjoint operator $\widetilde{L}$ of type I admits a factorization of the form

$$
\widetilde{L}=Q^{+} Q+v_{T},
$$

where the operator $Q^{+}$has the form (161), if and only if for every vertex $P$ the matrix $B_{P}=\left(b_{T: T^{\prime}}\right)$, where the black triangles $T$, $T^{\prime}$ belong to the star of the vertex $P$, has the form

$$
B_{P}=\operatorname{Diag}+\Lambda_{P}, \quad \operatorname{rk} \Lambda_{P}=1 .
$$

For multiplicities $N_{\mathrm{tr}}=4,6$ this condition is always satisfied. 
The operator $Q$ in (163) is defined up to a transformation that does not change any product of the form

$$
y_{T: P} y_{T^{\prime}: P} \quad\left(T \neq T^{\prime}\right) .
$$

In particular, for $N_{\mathrm{tr}} \geq 6$ the coefficients $y_{T: P}$ are defined uniquely, and for $N_{\mathrm{tr}}=4$ up to the transformation $\left(T \neq T^{\prime}\right)$

$$
y_{T: P} \mapsto \mu_{P} y_{T: P}, \quad y_{T^{\prime}: P} \mapsto \mu_{P}^{-1} y_{T^{\prime}: P},
$$

where $0 \neq \mu_{P}$ is any non-zero function from vertices with $N_{\operatorname{tr}}(P)=4$.

Theorem 155 A triangular (black) Schrödinger operator of type II always admits a factorization of the form

$$
L=Q Q^{+}+u_{T}
$$

where

$$
\left(Q^{+} \psi\right)_{T_{1}}=\sum_{T} y_{T_{1}: T} \psi_{T}+x_{T_{1}} \psi_{T_{1}},
$$

$T_{1}$ is a white triangle, and the sum is taken over all black triangles $T$ adjacent to $T_{1}$. In fact, the coefficients $y_{T_{1}: T}$ correspond to the edges of the triangulation.

We have the equalities

$$
y_{T: T_{1}} y_{T^{\prime}: T_{1}}=b_{T: T^{\prime}}
$$

for any three triangles $T, T_{1}, T^{\prime}$ sequentially adjacent to each other (black, white, black) from the star of a vertex with $N_{\mathrm{tr}} \geq 6$;

$$
y_{T: T_{1}} y_{T^{\prime}: T_{1}}+y_{T: T_{1}^{\prime}} y_{T^{\prime}: T_{1}^{\prime}}=b_{T: T^{\prime}}
$$

for black triangles $T, T^{\prime}$ and white triangles $T_{1}, T_{1}^{\prime}$ from the star of a vertex with $N_{\mathrm{tr}}=4$.

The factorization is unique in the neighbourhood of all vertices $P$ such that $N_{\mathrm{tr}}(P) \geq 6$. In the neighbourhood of vertices $P$ with $N_{\mathrm{tr}}(P)=4$ the factorization is not unique: every equation of the form (167) can be replaced by two:

$$
y_{T: T_{1}} y_{T^{\prime}: T_{1}}=b_{T: T^{\prime}}^{(1)}, \quad y_{T: T_{1}^{\prime}} y_{T^{\prime}: T_{1}^{\prime}}=b_{T: T^{\prime}}^{(2)}
$$

where $b_{T: T^{\prime}}=b_{T: T^{\prime}}^{(1)}+b_{T: T^{\prime}}^{(2)}$ is an arbitrary decomposition of $b_{T: T^{\prime}}$ into a sum of two non-zero terms. The resulting system is uniquely soluble.

In this theorem the black and white colours can be interchanged.

Remark 17 If for all vertices of the triangulation we have $N_{\mathrm{tr}}=4,6$, then the classes of operators of types I and II coincide. 
Corollary 5 1) If for all vertices we have $N_{\mathrm{tr}} \leq 6$ (that is, the curvature is non-negative), then factorizations of all types are defined. Correspondingly, all the Laplace transformations related to them are also defined. For example, for a vertex operator the Laplace transformation has the form

$$
L=Q Q^{+}+w \longmapsto \widetilde{L}=Q^{+} w^{-1} Q+1 .
$$

2) Factorizations of triangular Schrödinger operators L of type II are always defined in terms of triangles of the opposite colour. The degree of non-uniqueness of these factorizations and the corresponding Laplace transformations is determined by the number of vertices $P$ of positive curvature, $N_{\operatorname{tr}}(P)=4$.

Remark 18 For an equilateral triangular lattice in the plane the spaces of functions on vertices, on black and on white triangles, can be identified, and then these factorizations coincide (up to equivalence (104)) with the factorizations of operators on a regular lattice considered in $\S 6$.

Conclusion. Formulation of the problem on cyclic Laplace chains arises in two cases.

Case 1: for $N_{\text {tr }} \leq 6$ (Corollary 5).

Case 2: for vertices with $N_{\mathrm{tr}}=4$ under the conditions of Corollary 5 .

In case 2 we can limit ourself to Laplace transformations for operators of type II, which transform functions on black triangles into functions on white triangles and vice versa. This is similar to one-dimensional Darboux transformations.

\section{§9. Simplicial connections. Generalizations}

We consider a simplicial complex $K$. Everywhere we denote a simplex of dimension $l$ by $\sigma^{l}$.

Definition 15 A simplicial connection of type $(q, j, k), 0 \leq j<k$, is an equation

$$
\sum_{\sigma^{q} \subset \sigma^{q+k}} c_{\sigma^{q+k}: \sigma^{q}} \psi_{\sigma^{q}}=0
$$

determined by a vector-function which ascribes to every pair of simplexes $\sigma^{q}, \sigma^{q+k} \subset K$ such that $\sigma^{q} \subset \sigma^{q+k}$ a collection

$$
c_{\sigma^{q+k}: \sigma^{q}}=\left(c_{\sigma^{q+k}: \sigma^{q}}^{\alpha}\right) \in \mathbb{R}^{m}, \quad \alpha=1, \ldots, m,
$$

where

$$
m=C_{q+k+1}^{q+1}-C_{q+j+1}^{q+1} .
$$

We require that the function $c_{\sigma^{q+k}: \sigma^{q}}$ satisfies the conditions for non-degeneracy and localization (see below). 
Such a function defines an operator $Q^{+}$which transforms the space of numerical functions of simplexes $\sigma^{q}$ into the space of $m$-vector-functions of simplexes $\sigma^{q+k}$ :

$$
\left(Q^{+} \psi\right)_{\sigma^{q+k}}=\sum_{\sigma^{q}} c_{\sigma^{q+k}: \sigma^{q}} \psi_{\sigma^{q}} \in \mathbb{R}^{m} .
$$

Simplicial connection depends only on the zero-space of this operator:

$$
Q^{+\alpha} \psi=0, \quad \alpha=1, \ldots, m .
$$

In fact, this relation is written separately in each simplex $\sigma^{q+k}$.

Requirement of non-degeneracy. Equation (197) should be such that for any subsimplex $\sigma^{q+k} \subset K$ an arbitrarily given collection of values $\psi_{\sigma^{q}}$ in simplexes $\sigma^{q}$, forming a $q$-dimensional skeleton of any simplex $\sigma^{q+j} \subset \sigma^{q+k}$, should uniquely and consistently determine values of $\psi$ on all remaining $q$-dimensional subsimplexes in $\sigma^{q+k} \subset K$. Thus, we can arbitrarily define values of $\psi$ in the $q$-dimensional skeleton of simplexes $\sigma^{q+j} \subset \sigma^{q+k}$.

Lemma 200 Suppose we are given a path $\gamma$ composed of $(q+k)$-dimensional simplexes $\sigma_{1}^{q+k}, \sigma_{2}^{q+k}, \ldots$, where $\sigma_{s}^{q+k}$ and $\sigma_{s+1}^{q+k}$ intersect exactly along a face of dimension $q+j$ for all $s$. Then the simplicial connection consistently defines a solution of (170) along the path $\gamma$, starting from arbitrary initial data defined in a q-dimensional skeleton of an arbitrary $(q+j)$-dimensional face of any of the simplexes $\sigma_{s}^{q+k}$ that make up this path.

We obtain the proof of the lemma in a simple way from the definitions: a solution is constructed by transition from the simplex $\sigma_{s}^{q+k}$ to the simplex $\sigma_{s+1}^{q+k}, s=1,2, \ldots$.

The analogue of curvature arises naturally for 'closed' paths $\gamma$ in which $\sigma_{1}^{q+k}=\sigma_{N}^{q+k}$. In this case there arises a 'holonomy transformation' of the simplicial connection (170) along the path $\gamma$ : solving (170) along the path according to the lemma, starting from some face $\sigma_{0}^{q+j} \subset \sigma_{1}^{q+k}$, we finally find the value of $\psi$ on all $q$-dimensional faces of the simplex $\sigma_{N}^{q+k}$, including $\sigma^{q} \subset \sigma_{0}^{q+j}$. These values may not coincide with the initial values. There arises the linear transformation

$$
R_{\gamma} \mathbb{R}^{M} \rightarrow \mathbb{R}^{M}
$$

where $M=C_{q+j+1}^{q+1}$ is the number of $q$-dimensional faces in the simplex $\sigma_{0}^{q+j} \subset$ $\sigma_{1}^{q+k}$ on which the values of the function $\psi_{\sigma^{q}}$ were given arbitrarily.

Starting from the requirement of non-degeneracy we can reduce equation (170) in any simplex $\sigma^{q+k}$ to such a form that inverse operators are defined for any pair of simplexes $\sigma_{1}^{q+j}, \sigma_{2}^{q+j} \subset \sigma^{q+k}$ :

$$
L_{12} \mathbb{R}_{1}^{M} \rightarrow \mathbb{R}_{2}^{M}
$$

where the space $\mathbb{R}_{\varepsilon}^{M}$ consists of all possible values of $\psi$ on simplexes $\sigma^{q} \subset \sigma_{\varepsilon}^{q+j}$, $\varepsilon=1,2$, and the bases in them are $\delta$-functions of the simplexes $\sigma^{q}$. 
Requirement of localization. All operators $L_{12}$ are uniquely defined only by the minimal simplex containing $\sigma_{1}^{q+j}$ and $\sigma_{2}^{q+j}$ :

$$
\left(\sigma_{1}^{q+j} \cup \sigma_{2}^{q+j}\right) \subset \sigma^{q+j+s} \subseteq \sigma^{q+k} .
$$

This means that if two simplexes $\sigma_{1}^{q+k}$ and $\sigma_{2}^{q+k}$ intersect along the simplex $\sigma^{q+j+s}$, where $s>0$, then for them all the operators $L_{12}$ inside the simplex $\sigma^{q+j+s}$ coincide.

The requirment of localization is automatically satisfied in two cases.

Case 1. $k=j+1$. Here always $\sigma^{q+j+1}=\sigma^{q+k}$.

Case 2. In a simplicial complex $K$ the simplexes $\sigma_{1}^{q+k}$ and $\sigma_{2}^{q+k}$ coincide if they intersect in a face of dimension greater than $q+j$.

In the examples already considered in this work (above) we have always had $q=0$, that is, functions $\psi$ were defined on vertices. Localization corresponded to cases 1 or 2 .

A trivial example Let $k=1, q=0, j=0$. We have the usual Abelian connection, sitting on edges of the complex $K$. In fact, from (170)

$$
c_{\sigma^{1}: \sigma_{1}^{0}} \psi_{\sigma_{1}^{0}}+c_{\sigma^{1}: \sigma_{2}^{0}} \psi_{\sigma_{2}^{0}}=0
$$

we arrive in this case at the operator of multiplication

$$
L_{12}=-\frac{c_{\sigma^{1}: \sigma_{1}^{0}}}{c_{\sigma^{1}: \sigma_{2}^{0}}} \psi_{\sigma_{1}^{0}} \longmapsto \psi_{\sigma_{2}^{0}}=L_{12}\left(\psi_{\sigma_{1}^{0}}\right) .
$$

Example 13 a) Let $q=0, k=2, j=1$ and let $K$ be a triangulation of a twodimensional surface. Here we have one equation. This situation was studied above (see $\S 6$ ).

For $K=\mathbb{R}^{2}$ with an equilateral triangular lattice we paid particular attention to the case of 'zero local curvature' where a solution of the equation $Q^{+} \psi=0$ reduces to a difference equation of the second order on the straight line (see Example 11). According to a hypothesis of the authors, this situation arises in some interesting cases when the point $\lambda=0$ is supposedly the lower bound of the continuous spectrum of the Schrödinger operator (123). On surfaces with non-trivial topology the case of zero local curvature leads to the global monodromy defined on the group $\pi_{1}$.

b) Let $q=0, j=1, k=2$ and let $K$ be the 'black part' (or 'white part') of the black and white triangulation of a surface. Here we also have one equation $Q^{+} \psi=0$, but we do not have local curvature, since black triangles are adjacent to each other at vertices (there are no common edges). Therefore there are no non-trivial paths.

c) Let $q=0, j=0, k=2$, and let $K$ be the same as in b). Here we have curvature.

Example 14 Let $q=0, k=3$, and let $K$ be the lattice of regular tetrahedra in $\mathbb{R}^{3}$. We have $K=K_{1} \cup K_{2}$, where $K_{1}$ is the 'black' part, $K_{2}$ is the 'white' 
part, and $K_{1} \cap K_{2}$ is a one-dimensional skeleton. Adjacency of two tetrahedra in $K$ is possible along no more than an edge, and adjacency of tetrahedra inside $K_{1}$ (or $K_{2}$ ) is only possible at a vertex. We have already considered the following cases a), b), see $\S 7$ :

a) $j=2$, complex $K_{1}$ (or $K_{2}$ ). There is no local curvature here.

b) $j=2$, complex $K$. There is also no local curvature here, since there are no paths $\gamma$. Nevertheless, there is a non-trivial condition for consistency (153) which ensures an extensive space of solutions of the equation $Q^{+} \psi=0$. This particular analogue of curvature has not been studied in a general form.

c) $j=1$, complex $K$. Here the concept of local curvature arises, since we have many paths $\gamma$. The system (170) has a solution in the whole of $\mathbb{Z}^{3}$, which is uniquely determined by an arbitrary pair of values of $\psi$ at the vertices of any edge if and only if the following condition is satisfied: eight equations, corresponding to each set of four tetrahedra, a pair of black and a pair of white, from the star of one vertex, such that both black tetrahedra adjoin each of the white ones along an edge, are linearly dependent. This assertion means that our system in the star of every vertex has a two-dimensional local space of solutions. Geometrically, 'curvatures' that obstruct this solution correspond on the border of the star - a sphere $S^{2}$ divided into triangles and squares-just to squares. In all we have six conditions at each vertex (the number of squares), but one of them is dependent.

d) Let $q=0, j=0$, and let $K$ be $K_{1}$. There are paths here composed of tetrahedra, linked along vertices. The space $\mathbb{R}^{M}$ for $j=0$ is one-dimensional, and we arrive only at Abelian connections. This is true for $j=0$ in any simplicial complexes for any $q$.

Lemma 211 A simplicial connection of type $(q, j, k)$ that satisfies the conditions for non-degeneracy and localization defines a multiplicative curvature transformation

$$
R_{\gamma} \mathbb{R}^{M} \rightarrow \mathbb{R}^{M}, \quad R_{\gamma_{1} \gamma_{2}}=R_{\gamma_{1}} R_{\gamma_{2}}, \quad R_{\gamma^{-1}}=R_{\gamma}^{-1}
$$

for any path $\gamma$,

$$
\gamma=\sigma_{1}^{q+k} \sigma_{2}^{q+k} \cdots \sigma_{N}^{q+k}
$$

where $\sigma_{N}^{q+k}=\sigma_{1}^{q+k}$ and the intersection $\sigma_{s}^{q+k} \cap \sigma_{s+1}^{q+k}$ is a face of dimension greater than or equal to $q+j$ for all $s=1, \ldots, N$.

The proof of the lemma is easily obtained from the previous one.

We now consider real discrete self-adjoint operators

$$
(L \psi)_{\sigma_{2}^{q}}=\sum_{\sigma_{1}^{q}} b_{\sigma_{1}^{q}: \sigma_{2}^{q}} \psi_{\sigma_{2}^{q}},
$$

acting on functions of $q$-simplexes of the complex $K$. We consider only 'operators of the second order', where the coefficients $b_{\sigma_{1}^{q}: \sigma_{2}^{q}}$ are different from zero only for 'nearest neighbours'. 
Definition 16 The simplexes $\sigma_{1}^{q}, \sigma_{2}^{q}$ are $k_{+}$-nearest ( $k_{-}$-nearest) if this pair is contained in some simplex $\sigma^{q+k_{+}}$(respectively, if the intersection $\sigma_{1}^{q} \cap \sigma_{2}^{q}$ is a simplex $\sigma^{q-k_{-}}$).

We can represent the operator $L$ as the sum of operators of two types $L=$ $L_{+}+L_{-}$. We consider these types separately;

$$
\begin{aligned}
\left(L_{+} \psi\right)_{\sigma_{2}^{q}} & \left.=\sum_{\sigma_{1}^{q}, \sigma_{2}^{q} \subset \sigma^{q+k_{+}}} b_{\sigma_{2}^{q}: \sigma^{q+k_{+}}: \sigma_{1}^{q}} \psi_{\sigma_{1}^{q}}, \quad \text { (type }+\right) \\
\left(L_{-} \psi\right)_{\sigma_{4}^{q}} & \left.=\sum_{\sigma_{3}^{q} \cap \sigma_{4}^{q} \supset \sigma^{q-k_{-}}} b_{\sigma_{4}^{q}: \sigma^{q-k_{-}}: \sigma_{3}^{q}} \psi_{\sigma_{3}^{q}}, \quad \text { (type- }\right)
\end{aligned}
$$

where $\sigma_{1}^{q}, \sigma_{2}^{q}$ are $k_{+}$-nearest and $\sigma_{3}^{q}, \sigma_{4}^{q}$ are $k_{-}$-nearest. Let $k_{+}=k_{-}=k$. We consider an operator $Q^{+}$of the same type as in the definition of a simplicial connection, and the operator $Q_{1}$ :

$$
\left(Q^{+} \psi\right)_{\sigma^{q+k}}=\sum_{\sigma^{q}} c_{\sigma^{q+k}: \sigma^{q}} \psi_{\sigma^{q}}, \quad\left(Q_{1} \psi\right)_{\sigma^{q-k}}=\sum_{\sigma^{q}} c_{\sigma^{q}: \sigma^{q-k}} \psi_{\sigma^{q}}
$$

Here the images are vector-functions, and the $\psi$ are scalars.

Definition 17 1) A factorization of the first type is a representation of $L_{+}$in the form

$$
L_{+}=Q Q^{+}+w
$$

where $Q^{+}$is the operator adjoint to $Q$ and $w=w_{\sigma^{q}}$ is the operator of multiplication by a numerical function.

A special factorization of the first type is a representation (176), where $w=$ const.

2) A factorization of the second type is a representation of the operator $L_{-}$ in the form

$$
L_{-}=Q_{1}^{+} Q_{1}+v,
$$

where $v$ is the operator of multiplication by a function of $\sigma^{q}$ and the operator $Q_{1}^{+}$is adjoint to $Q_{1}$.

A special factorization of the second type is the case when $v=$ const.

3) A Laplace transformation of the operators $L_{ \pm}$is defined by the formulae

$$
\begin{aligned}
& L_{+} \longmapsto \widetilde{L}_{+}=Q^{+} w^{-1} Q+1, \\
& L_{-} \longmapsto \widetilde{L}_{-}=Q_{1} v^{-1} Q_{1}^{+}+1,
\end{aligned}
$$

and their zero eigenvectors are transformed by well-known formulae into the eigenvectors of $\widetilde{L}_{ \pm}$:

$$
\begin{array}{lll}
\psi^{+} \longmapsto \widetilde{\psi}^{+}=Q^{+} \psi^{+}, & \widetilde{L}_{+} \widetilde{\psi}^{+}=0, & L_{+} \psi^{+}=0, \\
\psi^{-} \longmapsto \widetilde{\psi}^{-}=Q_{1} \psi^{-}, & \widetilde{L}_{-} \widetilde{\psi}^{-}=0, & L_{-} \psi^{-}=0 .
\end{array}
$$


Obviously, the operator $\widetilde{L}_{+}$is written analogously to $L_{-}$, where $\widetilde{Q}^{+}=v^{-1 / 2} Q^{+}$. These formulae assume that $w \geq 0, v \geq 0$, although this does not play a serious role here.

Detailed studies have been devoted above to the investigation of factorizations and Laplace transformations in particular cases. Here we consider only the simplest example, that is, a standard discrete real Schrödinger operator.

Example 15 Let $q=0, k=1, j=0$. We have an operator defined on functions of vertices

$$
L=L_{+}, \quad(L \psi)_{\sigma_{2}^{0}}=\sum_{\sigma_{1}^{0} \cup \sigma_{2}^{0}=\partial \sigma^{1}} b_{\sigma_{2}^{0}: \sigma^{1}: \sigma_{1}^{0}} \psi_{\sigma_{1}^{0}}+b_{\sigma_{1}^{0}} \delta\left(\sigma_{2}^{0}, \sigma_{1}^{0}\right) \psi_{\sigma_{1}^{0}} .
$$

(The last term is present only for $\sigma_{2}^{0}=\sigma_{1}^{0}$; this is shown by the $\delta$-function.)

We can always factorize such an operator in the form (we always suppose that $b_{\sigma_{2}^{0}: \sigma^{1}: \sigma_{1}^{0}} \neq 0$ )

$$
L=Q Q^{+}+w,
$$

where $w$ is some function. This factorization is not unique.

Question Is there always a 'special factorization', where $w=$ const? It exists for the complex $K=\mathbb{Z}^{1}$, see $\S 3$, but in the general case the answer is not clear (we assume that $b_{\sigma^{0}} \geq 0$ ).

Remark 19 Earlier (see $\S 8$ ) in connection with the Laplace transformation on surfaces with black-white triangulation we considered yet another form of the factorization: let the complex $K$ and two subcomplexes $K_{1}, K_{2}$ be given, where $K_{1} \cap K_{2}$ is a $(q-1)$-dimensional skeleton of the complex $K$, and the operator $L$ acts on functions of $\sigma^{q} \subset K_{1}$ (white $q$-simplexes):

$$
(L \psi)_{\sigma_{2}^{q}}=\sum_{\sigma_{1}^{q}} b_{\sigma_{1}^{q}: \sigma_{2}^{q}} \psi_{\sigma_{1}^{q}}, \quad \sigma_{1}^{q}, \sigma_{2}^{q} \subset K_{1} .
$$

The notion of the 'nearest $k$-neighbourhood' is defined for simplexes from $K_{1}$ by means of faces of dimension $q-k$, which are common for them with an arbitrary 'black' $q$-simplex from $K_{2}$. The factorization is sought in the form (179), where $w$ is the operator of multiplication by a function, and $Q^{+}$is an operator of the form

$$
\left(Q^{+} \psi\right)_{\bar{\sigma}^{q}}=\sum c_{\bar{\sigma}^{q}: \sigma^{q-k}: \sigma^{q}} \psi_{\sigma^{q}},
$$

where $\bar{\sigma}^{q} \subset K_{2}, \sigma^{q} \subset K_{1}, \sigma^{q-k} \subset \bar{\sigma}^{q} \cap \sigma^{q}$. Analogously, the operators $\bar{L}$ act on 'black' $q$-simplexes $\bar{\sigma}^{q} \subset K_{2}$ and the factorization is sought in the form

$$
\bar{L}=Q^{+} Q+v .
$$

Previous formulae define Laplace transformations and also special Laplace transformations if the potentials $v, w$ are constant. 
Example 16 Let $K$ be a $q$-dimensional triangulated manifold with blackwhite coloured $q$-dimensional simplexes, $K=K_{1} \cup K_{2}$, and let $K_{1} \cap K_{2}$ be a $(q-1)$-dimensional skeleton. For $k=1$ we have $\sigma^{q-1}$ as a common face of exactly two simplexes $\bar{\sigma}^{q}, \sigma^{q}$, that is, black and white. By analogy with the case $q=2$ (see $\S 8$ ), the condition for factorization depends on the $N\left(\sigma^{q-2}\right)$ multiplicity of $(q-2)$-dimensional simplexes $\sigma^{q-2} \subset K$, which is equal to the number of adjacent $(q-1)$-dimensional $\sigma^{q-1} \supset \sigma^{q-2}$. This number is even, $N \geq 4$. For $\sigma_{j}^{q} \subset K_{1}, \bar{\sigma}_{j}^{q} \subset K_{2}$ we have:

a) $b_{\sigma_{1}^{q}: \sigma_{2}^{q}}=c_{\sigma_{1}^{q}: \sigma_{1}^{q-1}: \bar{\sigma}^{q}} c_{\sigma_{2}^{q}: \sigma_{2}^{q-1}: \bar{\sigma}^{q}}, \quad \sigma^{q-2}=\sigma_{1}^{q-1} \cap \sigma_{2}^{q-1}, \quad N\left(\sigma^{q-2}\right) \geq 6$;

b) $b_{\sigma_{1}^{q}: \sigma_{2}^{q}}=c_{\sigma_{1}^{q}: \sigma_{1}^{q-1}: \bar{\sigma}_{1}^{q}} c_{\sigma_{2}^{q}: \sigma_{2}^{q-1}: \bar{\sigma}_{1}^{q}}+c_{\sigma_{1}^{q}: \sigma_{3}^{q-1}: \bar{\sigma}_{2}^{q}} c_{\sigma_{2}^{q}: \sigma_{4}^{q-1}: \bar{\sigma}_{2}^{q}}, \quad \sigma^{q-2}=\sigma_{1}^{q-1} \cap$ $\sigma_{2}^{q-1} \cap \sigma_{3}^{q-1} \cap \sigma_{4}^{q-1}, \quad N\left(\sigma^{q-2}\right)=4$.

Let us consider a): the number of equations is equal to the number of $(q-2)$-dimensional faces of the simplex $\bar{\sigma}^{q}$, that is, $l=q(q+1) / 2$. The number of unknowns is $m(q+1)$, where $m$ is the dimension of the vector $c_{\sigma^{a}: \sigma^{q-1}: \bar{\sigma}^{q}}$. Thus we have the condition $m(q+1) \geq q(q+1) / 2$ or $m \geq q / 2$. The scalar factorization $(m=1)$ needs to satisfy additional conditions for $q \geq 3$.

Example 17 We again consider $K$, a $(q+2)$-dimensional manifold with 'blackwhite' coloured ( $q+2)$-simplexes, $K=K_{1} \cup K_{2}$, and $K_{1} \cap K_{2}$, a $(q+1)$-skeleton. Suppose we are given an operator of some other type, acting on functions of $q$-simplexes from $K$

$$
(L \psi)_{\sigma_{2}^{q}}=\sum_{\sigma_{1}^{q}} b_{\sigma_{2}^{q}: \sigma_{1}^{q}} \psi_{\sigma_{1}^{q}},
$$

and we seek a 'white' factorization (179), where $w$ is the operator of multiplication by a function, and

$$
\left(Q^{+} \psi\right)_{\sigma^{q+2}}=\sum_{\sigma^{q}} c_{\sigma^{q+2}: \sigma^{q}} \psi_{\sigma^{q}}, \quad \sigma^{q+2} \subset K .
$$

We arrive at the relation

$$
b_{\sigma_{1}^{q}: \sigma_{2}^{q}}=c_{\sigma^{q+2}: \sigma_{1}^{q}} c_{\sigma^{q+2}: \sigma_{2}^{q}} .
$$

The number of unknowns is equal to $m(q+2)(q+3) / 2=\operatorname{lm}$ (if $c_{\sigma^{q+2}: \sigma_{1}^{q}}$ is an $m$-vector) and the number of equations is equal to $l(l-1) / 2$. The factorization condition thus has the form

$$
l m \geq l(l-1) / 2,
$$

$(q+2)(q+3) / 2=l, m \geq(l-1) / 2$.

For $q=1, k=2$ we see that $m=3$ or 5 is acceptable. In these cases factorization is always possible in the form (179). The possibility of the special factorization $w=$ const is of interest: one would like to clarify this question. If the factorization is special, then the question of zero modes of the 
operator $L=Q Q^{+}$is of interest, that is, the question of the solubility in the space $\psi \in \mathcal{L}_{2}$ of the equation $Q^{+} \psi=0$. For $m=3$ and $m=5$ equations of this type determine the connections (if the conditions for non-degeneracy and localization are satisfied). Paths along which 'parallel transport' is realized and curvature is defined consist of 3 -simplexes $(q=1)$ adjoining each other along edges $(m=5)$ or along faces $(m=3)$. The requirement of localization is automatically satisfied for the case $m=5, K=K_{1}$ (white tetrahedra).

If $m=3$, then purely white paths do not exist. The curvature is defined by the pair of equations: $Q_{1}^{+} \psi=0$ (white part), $Q_{2}^{+} \psi=0$ (black part), that is, $K=K_{1} \cup K_{2}$.

Now suppose we are given an arbitrary simplicial connection on a complex $K$ of type $(q, j, k)$.

Definition 18 The local curvature of the vertex $\sigma^{0} \subset K$ is the curvature of this connection on special paths of the subcomplex $K_{P}$, which is the simplicial star of the vertex $\sigma^{0}$ : we take all simplexes $\sigma_{c}^{q}$ with vertex $\sigma^{0}, \sigma^{q} \subset K_{P}$, paths of the form

$$
\gamma=\left(\sigma_{1}^{q+k}, \sigma_{2}^{q+k}, \ldots, \sigma_{N}^{q+k}=\sigma_{1}^{q+k}\right),
$$

such that $\sigma_{j}^{q+k}=\sigma^{0} \sigma_{j}^{q-1+k}$.

A transformation $R_{\gamma}$ determined only by paths $\gamma \subset K_{P}$ (182) is called the local curvature of the simplicial connection at the point $\sigma^{0} \subset K$.

Hypothesis For manifolds $K$ the curvature is trivial if the local curvatures of all the vertices are trivial (we note that in a number of cases above $K$ was not a manifold).

\section{References}

[1] I. A. Dynnikov and S. P. Novikov. Laplace transformations and simplicial connections. Uspekhi Mat. Nauk, 1997, 52. no. 6, 157-158. English transl. in Russian Math. Surveys, 52 (1997), 6.

[2] B. A. Dubrovin, V. B. Matveev, and S. P. Novikov. Non-linear equations of the Korteweg-de Vries type, finite zone linear operators, and Abelian varieties. Uspekhi Mat. Nauk, 31 (1976), 1, 55-136. English transl. in Russian Math. Surveys, 31 (1976), 1.

[3] V. E. Zakharov, S. V. Manakov, S. P. Novikov, and L. P. Pitaevskii. Theory of solitons. Moscow 1980, Nauka. (Russian).

[4] E. A. Kuznetsov and A. V. Mikhailov. Stability of stationary waves in non-linear weakly dispersive media. Zh. Eksper. Teoret. Fiz., 67 (1974), 1717-1727. English transl. in Soviet Phys. JETP, 40 (1975). 
[5] I. M. Krichever. Potentials with zero coefficient of reflection against a background of finite-zone potentials. Funktsional. Anal. i Prilozhen., 9 (1975), 2, 77-78. English transl. in Functional Anal. Appl., 9 (1975).

[6] S. P. Novikov. A periodic problem of the Korteweg-de Vries equation. I. Funktsional. Anal. i Prilozhen, 8 (1974), 3, 54-66. English transl. in Functional Anal. Appl., 8 (1974).

[7] B. A. Dubrovin and S. P. Novikov. Ground states in a periodic field. Magnetic Bloch functions and vector bundles. Dokl. Akad. Nauk SSSR, 253 (1980), 1293-1297. English transl. in Soviet Math. Dokl., 22 (1980).

[8] B. A. Dubrovin and S. P. Novikov. Ground states of a two-dimensional electron in a periodic magnetic field. Zh. Eksper. Teoret. Fiz., 79 (1980), 1006-1016. English transl. in Soviet Phys. JETP, 52 (1980).

[9] A. P. Its and V. B. Matveev. Schrödinger operator with a finite-zone spectrum and $N$-soliton solutions of the Korteweg-de Vries equation. Teoret. Mat. Fiz., 23 (1975), 51-68. English transl. in Theoret. and Math. Phys. 23 (1975).

[10] P. Lax. Periodic solutions of the Korteweg-de Vries equation. Comm. Pure Appl. Math., 28 (1975), 141-188.

[11] H. McKean and P. Van Mörbeke. The spectrum of Hill's equation. Invent. Math., 30 (1975), 217-274.

[12] J. Weiss. Periodic fixed points of Bäcklund transformations and the KdV equation. J. Math. Phys., 27 (1986), 2647-2656.

[13] A. P. Veselov and A. B. Shabat. A dressing chain and the spectral theory of the Schrödinger operator. Funktsional. Anal. i Prilozhen., 27 (1993), 2, 1-21. English transl. in Functional Anal. Appl., 27 (1993).

[14] V. B. Matveev and M. A. Salle. Darboux transformations and solitons, Springer-Verlag, Berlin 1991.

[15] S. P. Novikov. Two-dimensional Schrödinger operators in periodic fields. In: Current problems in mathematics, Vol. 23, VINITI, Moscow 1983, 3-32.

[16] I. M. Krichever. Algebro-geometric construction of the Zakharov-Shabat equations and their periodic solutions. Dokl. Akad. Nauk SSSR, 227 (1976), 291-294. English transl. in Soviet Math. Dokl., 17 (1976).

[17] I. M. Krichever. Methods of algebraic geometry in the theory of non-linear equations. Uspekhi Mat. Nauk, 32 (1977), 6, 183-208. English transl. in Russian Math. Surveys, 32 (1977), 6.

[18] H. Flaschka. On the Toda lattice,. II. Inverse-scattering solutions. Progr. Theoret. Phys., 51 (1974), 703-716. 
[19] S. V. Manakov. Complete integrability and stochastization in discrete dynamical systems. Zh. Eksper. Teoret. Fiz., 67 (1974), 543-555. English transl. in Soviet Phys. JETP, 40 (1975).

[20] B. A. Dubrovin, I. M. Krichever, and S. P. Novikov Integrable systems. I. in: Current problems in mathematics. Fundamental directions, Vol. 4, VINITI, Moscow 1985, 179-285.

[21] V. Spiridonov, L. Vinet, and A. Zhedanov. Difference Schrödinger operators with linear and exponential discrete spectra. Lett. Math. Phys., 29 (1993), 63-73.

[22] S. P. Novikov and I. A. Taimanov. Difference analogs of the harmonic oscillator. Appendix II in [31].

[23] N. M. Atakishiev and S. K. Suslov. Difference analogues of the harmonic oscillator. Teor. Mat. Fiz., 85 (1990), 64-73. English transl. in Theoret. and Math. Phys. 85 (1990).

[24] S. P. Finikov. Theory of congruences. Moscow-Leningrad 1950, Gostekhizdat. (Russian).

[25] G. Darboux. Leçons sur la théorie générale des surfaces et des applications géométriques du calcul infinitésimal. Paris 1887-1896, Gauthier-Villars.

[26] G. Tzitzéica. Géométrie différentielle projective des réseaux. ParisBucharest 1924.

[27] A. V. Mikhailov. Integrability of a two-dimensional generalization of the Toda chain. Pis'ma v Zh. Eksper. Teoret. Fiz., 30 (1979), 7, 443-448. English transl. in JETP Letters, 30 (1979).

[28] A. N. Leznov and M. V. Savel'ev. Group methods of integrability of nonlinear dynamical systems. Nauka, Moscow 1985. (Russian).

[29] S. Bulgadaev. Two-dimensional integrable field theories connected with simple Lie algebras. Phys. Lett., 96B (1980), 151-153.

[30] A. P. Veselov and S. P. Novikov. Exactly soluble two-dimensional Schrödinger operators. Uspekhi Mat. Nauk, 50 (1995), 6, 171-172. English transl. in Russian Math. Surveys, 50 (1995), 6.

[31] S. P. Novikov and A. P. Veselov. Exactly solvable two-dimensional Schrödinger operators and Laplace transformations. In: Solitons, geometry, and topology: on the crossroad, ed. V. M. Buchstaber and S. P. Novikov. AMS Transl. (2) 179 (1997), 109-132.

[32] B. A. Dubrovin, I. M. Krichever, and S. P. Novikov. The Schrödinger equation in a periodic field and Riemann surfaces. Dokl. Akad. Nauk SSSR, 229 (1976), 15-18. English transl. in Soviet Math. Dokl., 17 (1976). 
[33] A. P. Veselov and S. P. Novikov. Finite-zone, two-dimensional Schrödinger operators. Potential operators. Dokl. Akad. Nauk SSSR, 279 (1984), 784788. English transl. in Soviet Math. Dokl., 30 (1984).

[34] S. P. Novikov and A. P. Veselov. Two-dimensional Schrödinger operator: inverse scattering and evolutional equations. Physica D 18 (1986), 267-273.

[35] A. I. Bobenko. Constant mean curvature surfaces and integrable equations. Uspekhi Mat. Nauk, 46 (1991), 4, 3-42. English transl. in Russian Math. Surveys, 46 (1991), 4.

[36] Y. Aharonov and A. Casher. Ground state of a spin 1/2 charged particle. Phys. Rev. A, 19 (1979), 2461-2463.

[37] H. J. de Vega and F. A. Schaposnik. Classical vortex solution of the Abelian Higgs model. Phys. Rev. D, 14 (1976), 1100-1106.

[38] S. P. Novikov. Magnetic Bloch functions and vector bundles. Typical dispersion laws and their quantum numbers. Dokl. Akad. Nauk SSSR, 257 (1981), 538-543. English transl. in Soviet Math. Dokl., 23 (1981).

[39] A. S. Lyskova. On the Schrödinger operator in a magnetic field. Uspekhi Mat. Nauk, 36 (1981), 2, 189-190. English transl. in Russian Math. Surveys, 36 (1981), 2.

[40] I. M. Krichever. Two-dimensional periodic difference operators and algebraic geometry. Dokl. Akad. Nauk SSSR, 285 (1985), 31-36. English transl. in Soviet Math. Dokl. 32 (1985).

[41] I. Krichever, O. Lipan, P. Wiegman, and A. Zabrodin. Quantum integrable systems and elliptic solutions of classical discrete nonlinear equations. Preprint.

[42] S. P. Novikov. Difference analogs of Laplace transformations and twodimensional Toda lattices. Appendix I in [31]

[43] S. P. Novikov. Algebraic properties of two-dimensional difference operators. Uspekhi Mat. Nauk, 52 (1997), 1, 225-226. English translation in Russian Math. Surveys 52 (1997), 1.

[44] S. P. Novikov. Difference 2D Schrödinger operators: algebraic symmetry and exact solvability. Preprint, Univ. Paris-VII, Paris, April 1997. 